\title{
Volume of the set of locally diagonalizable bipartite states
}

\author{
Lin Zhang ${ }^{1 *}$ Seunghun Hong ${ }^{2 \dagger}$ \\ ${ }^{1}$ Institute of Mathematics, Hangzhou Dianzi University, Hangzhou 310018, PR China \\ ${ }^{2}$ Northwestern College, 101 7th St SW, Orange City, 51041 IA, USA
}

\begin{abstract}
The purpose of this article is to investigate the geometry of the set of locally diagonalizable bipartite quantum states. We have the following new results: the Hilbert-Schmidt volume of all locally diagonalizable states, and a necessary and sufficient condition for local diagonalizability in the qubit-qubit case. Besides, we partition the set of all locally diagonalizable states as local unitary orbits (or coadjoint orbits) of diagonal forms. It is well-known that the Riemannian volume of a coadjoint orbit for a regular point in a specified Weyl chamber can be calculated by Harish-Chandra's volume formula. By modifying HarishChandra's volume formula, we give, for the first time, a specific formula for the Riemannian volume of a local unitary orbit of a regular point in a specified Weyl chamber. Several open questions are presented as well.
\end{abstract}

Keywords: Euclid volume; Hilbert-Schmidt measure; Harish-Chandra's volume formula; local unitary orbit

\section{Introduction}

Qubits and qubit quantum channels are the simplest building blocks for quantum information processing and quantum computations. A qubit is the quantum analog of the classical bit; a qubit quantum channel is just the quantum analog of the transition probability matrix. Recently, Lovas and Andai [14] analyzed the structure of these qubit channels using the duality between quantum maps and quantum states, i.e., via Choi-Jamiłkowski correspondence [22]. They calculate the (Euclid) volume of general and unital qubit channels (real and complex case) with respect to the Lebesgue measure. For unital qubit channels, they are essentially equivalent to two-qubit states with the same completely-mixed marginal states via ChoiJamiłkowski representation.

In the recent decades, the geometric separability probability of bipartite systems, i.e., the ratio of volumes of the set of separable bipartite states to the set of all bipartite states on the same tensor space of two Hilbert spaces, has been extensively studied. In 1998, Życzkowski et al raised that question and gave

\footnotetext{
*E-mail: godyalin@163.com; linyz@hdu.edu.cn

$\dagger$ E-mail: seunghun.hong@gmail.com
} 
a detailed discussion about it [21]. To solve the problem, as suggested by the definition of separability probability, we need to calculate two volumes: (1) the volume of the set of all states acting on the same Hilbert space and (2) the volume of the set of all separable states acting on the bipartite Hilbert spaces. Luckily, the volume of the set of all states with respect to the Hilbert-Schmidt measure was computed by Życzkowski and Sommers [24] and Andai [1]. A review with detailed reasoning for such volume formula can be found in Zhang [20]. Computing the separability probability of two-qubit quantum states relative to the Hilbert-Schmidt measure is one of the simplest yet challenging problems. Numerical simulations lead to intriguing formulas for separability probability, presented in 2013 by Slater [19]: the separability probability for real two-qubit state is

$$
\frac{\operatorname{vol}_{\mathrm{HS}}\left(\mathrm{D}_{\mathrm{sep}}\left(\mathbb{R}^{2} \otimes \mathbb{R}^{2}\right)\right)}{\operatorname{vol}_{\mathrm{HS}}\left(\mathrm{D}\left(\mathbb{R}^{2} \otimes \mathbb{R}^{2}\right)\right)}=\frac{29}{64}
$$

and, for complex two-qubit it is

$$
\frac{\operatorname{vol}_{\mathrm{HS}}\left(\mathrm{D}_{\mathrm{sep}}\left(\mathbb{C}^{2} \otimes \mathbb{C}^{2}\right)\right)}{\operatorname{vol}_{\mathrm{HS}}\left(\mathrm{D}\left(\mathbb{C}^{2} \otimes \mathbb{C}^{2}\right)\right)}=\frac{8}{33},
$$

where $\mathrm{D}\left(\mathbb{K}^{m} \otimes \mathbb{K}^{n}\right)$ stands for the set of all bipartite density matrices acting on $\mathbb{K}^{m} \otimes \mathbb{K}^{n}$, and $\mathrm{D}_{\text {sep }}\left(\mathbb{K}^{m} \otimes \mathbb{K}^{n}\right)$ is the set of separable matrices in $\mathrm{D}\left(\mathbb{K}^{m} \otimes \mathbb{K}^{n}\right)$. Here $\mathbb{K}$ equals $\mathbb{R}$ or $\mathbb{C}$. The real case has been proved by Lovas and Andai [15]. But the complex case is still open at present. There are, however, results of Aubrun, Szarek, and Ye for the asymptotic separability probability in the limit of the dimension of the state system tending to infinity $[2,3]$, which are very interesting from a mathematical point of view, complementing the conjectured exact formulas in the low dimensional setting.

The purpose of this article is to infer some information about the set of bipartite quantum states that are locally diagonalizable. We have the following new results: a necessary and sufficient condition for local diagonalizability in the qubit-qubit case (Theorem 2.4) and the Hilbert-Schmidt volume of all locally diagonalizable states (Theorem 2.5). The celebrated Harish-Chandra's volume formula allows us to calculate the volume of a coadjoint orbit for a regular point in a specified Weyl chamber. By modifying Harish-Chandra's formula, we give, for the first time, a specific formula for the volume of a coadjoint local unitary orbit of a regular point in a specified Weyl chamber. As an application, we calculate the volume of the set of all bipartite quantum states that are locally unitary equivalent to a diagonal quantum state.

Here is the outline of the paper: After introducing basic notions that we use, we derive, in Section 2, the joint probability distribution density of all eigenvalues of all locally diagonalizable bipartite states. We also present a necessary and sufficient condition for a two-qubit to be locally unitary (LU) equivalent to a diagonal form. We then proceed to calculate the Hilbert-Schmidt (HS) volume of locally diagonalizable states (Theorem 2.5). In Section 3, we apply Harish-Chandra's volume formula to specific cases such as the unitary group and the tensor product of two unitary groups. Two main results of the Section are Theorems 3.5 and 3.8; the first yields an analytical formula for the volume of the tensor product of two unitary groups, and the second leads to the conclusion that the volume of a locally unitary orbit in the tensor product case equals the product of the volumes of the factors. We conclude in Section 4 with discussion and several open problems. 


\section{Volume of the set of all locally diagonalizable states}

Suppose we have two finite-dimensional Hilbert spaces $\mathcal{H}$ and $\mathcal{K}$. Specifically, let $\mathcal{H}=\mathbb{C}^{m}$ and $\mathcal{K}=\mathbb{C}^{n}$. Chose the standard basis $\{|i\rangle\}_{i=1}^{m}$ and $\{|j\rangle\}_{j=1}^{n}$ for $\mathbb{C}^{m}$ and $\mathbb{C}^{n}$, respectively. A qudit is represented by a positive semi-definite matrix of unit trace and we shall identify the two. Denote by $\mathrm{D}\left(\mathbb{C}^{k}\right)$ the set of all $k \times k$ density matrices. Then the set of all bipartite quantum states is $\mathrm{D}\left(\mathbb{C}^{m} \otimes \mathbb{C}^{n}\right)$. Throughout this paper, we do not distinguish the meaning of a state and a density matrix. A bipartite state $\rho_{A B}$ is separable if it is a probabilistic mixture of product states $\rho_{\mu}^{A} \otimes \rho_{\mu}^{B}$ where $\rho_{\mu}^{A} \in \mathrm{D}\left(\mathbb{C}^{m}\right)$ and $\rho_{\mu}^{B} \in \mathrm{D}\left(\mathbb{C}^{n}\right)$. In other words, $\rho_{A B}$ is separable if

$$
\rho_{A B}=\sum_{\mu} p_{\mu} \rho_{\mu}^{A} \otimes \rho_{\mu}^{B}
$$

where $\left\{p_{\mu}\right\}_{\mu}$ is a probability distribution. If a bipartite state is not separable then it is said to be entangled. Thanks to the Spectral Decomposition Theorem, which says that the orbit of a Hermitian matrix under the adjoint action of unitary matrices contains a diagonal matrix, we see that a bipartite state $\rho_{A B} \in$ $\mathrm{D}\left(\mathbb{C}^{m} \otimes \mathbb{C}^{n}\right)$ can be diagonalized by a global unitary matrix $U \in \mathrm{U}(m n)$, where $\mathrm{U}(m n)$ can be understood as the unitary group comprising all unitary matrices on $\mathbb{C}^{m} \otimes \mathbb{C}^{n}$; thus,

$$
\rho_{A B}=U \Lambda U^{\dagger}, \quad \Lambda=\sum_{i=1}^{m} \sum_{j=1}^{n} \lambda_{i, j}|i, j\rangle\langle i, j|,
$$

where + denotes the adjoint. Note that all eigenvalues $\lambda_{i j}$ of $\rho_{A B}$ are indexed by two indices.

A bipartite state $\rho_{A B}$ is said to be locally diagonalizable if it is diagonalizable in the following manner:

$$
\rho_{A B}=\left(U_{A} \otimes U_{B}\right) \Lambda\left(U_{A} \otimes U_{B}\right)^{+}
$$

for a simple tensor $\left.U_{A} \otimes U_{B} \in \mathrm{U}(m) \otimes \mathrm{U}(n)\right)$; we may, in fact, require that $U_{A} \otimes U_{B} \in \mathrm{SU}(m) \otimes \mathrm{SU}(n)$. We denote the set of all locally diagonalizable bipartite states from $\mathrm{D}\left(\mathbb{C}^{m} \otimes \mathbb{C}^{n}\right)$ by the following notation:

$$
\mathscr{D}_{\mathrm{LU}}\left(\mathbb{C}^{m} \otimes \mathbb{C}^{n}\right):=\left\{\rho \in \mathrm{D}\left(\mathbb{C}^{m} \otimes \mathbb{C}^{n}\right): \rho \text { is locally diagonalizable }\right\} \text {. }
$$

Locally diagonalizability is intimately related to what is known as local unitary equivalence; two bipartite states $\rho$ and $\rho^{\prime}$ in $\mathrm{D}\left(\mathbb{C}^{m} \otimes \mathbb{C}^{n}\right)$ are said to be locally unitary $(L U)$ equivalent if

$$
\rho^{\prime}=(U \otimes V) \rho(U \otimes V)^{\dagger}
$$

for some simple tensor $U \otimes V \in \mathrm{SU}(m) \otimes \mathrm{SU}(n)$. So a bipartite state is locally diagonalizable if and only if it is LU equivalent to a diagonal state.

Not every bipartite state can be locally diagonalizable, and even so for separable states. This can be seen from simple dimension counting [4]. It is easily seen that $\operatorname{dim}\left(\mathrm{D}\left(\mathbb{C}^{m} \otimes \mathbb{C}^{n}\right)\right)=(m n)^{2}-1$. For the submanifold $\mathscr{D}_{\mathrm{LU}}\left(\mathbb{C}^{m} \otimes \mathbb{C}^{n}\right)$ we have the following identification:

$$
\begin{aligned}
\mathscr{D}_{\mathrm{LU}}\left(\mathbb{C}^{m} \otimes \mathbb{C}^{n}\right) & \simeq(\mathrm{U}(m) \otimes \mathrm{U}(n)) /\left(T_{(m)} \otimes T_{(n)}\right) \times \Delta_{m n-1} \\
& \simeq\left(\mathrm{U}(m) / T_{(m)}\right) \otimes\left(\mathrm{U}(n) / T_{(n)}\right) \times \Delta_{m n-1},
\end{aligned}
$$


where $\Delta_{k-1}:=\left\{\left(p_{1}, \ldots, p_{k}\right) \in \mathbb{R}_{+}^{k}: \sum_{j=1}^{k} p_{j}=1\right\}$ is the $(k-1)$-dimensional probability simplex, and $T_{(k)}$ denotes the (standard) maximal tori of the compact Lie group $\mathrm{U}(k)$ (more on this in Section 3). Therefore

$$
\operatorname{dim}\left(\mathscr{D}_{\mathrm{LU}}\left(\mathbb{C}^{m} \otimes \mathbb{C}^{n}\right)\right)=\left(m^{2}-1\right)+\left(n^{2}-1\right)+(m-1)(n-1) .
$$

We note that the minimum of $\operatorname{dim}\left(\mathscr{D}_{\mathrm{LU}}\left(\mathbb{C}^{m} \otimes \mathbb{C}^{n}\right)\right)$ for fixed $d:=m n$ is achieved at $m=n=\sqrt{d}$, while the maximum is achieved at $m=1$ or $n=1$. We also note that the set of all product mixed states form an $\left(m^{2}+n^{2}-2\right)$-dimensional subset of $\mathscr{D}_{\mathrm{LU}}\left(\mathbb{C}^{m} \otimes \mathbb{C}^{n}\right)$.

Before proceeding further, a few words on the notion of the volume of a smooth manifold is in order (for details, we refer to [8, Sec. 3.13]). Recall that an $n$-dimensional oriented manifold $M$ with a pseudo-Riemannian metric $g$ has a standard volume form $\omega$, known as the Riemannian volume form, whose expression in an oriented chart $\left(x^{1}, \ldots, x^{n}\right)$ is given by

$$
\omega=\sqrt{\operatorname{det}(g)} \mathrm{d} x^{1} \wedge \cdots \wedge \mathrm{d} x^{n} .
$$

If $D$ is a domain of integration in $\boldsymbol{M}$, then

$$
\operatorname{vol}_{g}(D):=\int_{D} \omega
$$

is called the Riemannian volume of $D$.

Of special interest is the case where $M$ is the set of all non-degenerate full-ranked density matrices from $\mathrm{D}\left(\mathbb{C}^{d}\right)$. (It is well-known that degenerate density matrices in $\mathrm{D}\left(\mathbb{C}^{d}\right)$ form a subset of zero-measure.) On $\mathrm{D}\left(\mathbb{C}^{d}\right)$ we have the Hilbert-Schmidt inner product, which is defined by

$$
\langle X, Y\rangle:=\operatorname{Tr}\left(X^{\dagger} Y\right)
$$

Differentiating this inner product yields a metric on $\boldsymbol{M}$ which we denote by $g_{\mathrm{HS}}$. We shall denote by $\mathrm{vol}_{\mathrm{HS}}$ the Riemannian volume form associated with $g_{\mathrm{HS}}$ and refer to the volume measured by $\mathrm{vol}_{\mathrm{HS}}$ as the Hilbert-Schmidt (HS) volume. Because the Hilbert-Schmidt inner product is invariant under the adjoint action, the induced metric and the associated Riemannian volume form are invariant. So the measure on $\mathrm{D}\left(\mathbb{C}^{d}\right)$ induced by $\mathrm{vol}_{\mathrm{HS}}$ is a constant multiple of the normalized Haar measure.

In order to compute the HS volume of all locally diagonalizable bipartite states, we wish to parametrize such states. Eigenvalues can serve that purpose, and knowing the density of eigenvalues essentially solves the question of finding the HS volume. The following lemma provides that density:

Lemma 2.1. The joint probability density of eigenvalues of all locally diagonalizable bipartite states in $\mathscr{D}_{\mathrm{LU}}\left(\mathbb{C}^{m} \otimes\right.$ $\left.\mathbb{C}^{n}\right)$ is given by

$$
\mathbf{P}(\Lambda) \propto\left[\prod_{1 \leqslant i<i^{\prime} \leqslant m} \sum_{j=1}^{n}\left(\lambda_{i j}-\lambda_{i^{\prime} j}\right)^{2}\right]\left[\prod_{1 \leqslant j<j^{\prime} \leqslant n} \sum_{i=1}^{m}\left(\lambda_{i j}-\lambda_{i j^{\prime}}\right)^{2}\right][\mathrm{d} \Lambda],
$$

where $[\mathrm{d} \Lambda]:=\prod_{i=1}^{m} \prod_{j=1}^{n} \mathrm{~d} \lambda_{i j}$ is the Lebesgue volume element for the diagonal matrix $\Lambda=\sum_{i=1}^{m} \sum_{j=1}^{n} \lambda_{i, j}|i, j\rangle\langle i, j|$. Proof. Let $\rho \in \mathscr{D}_{\mathrm{LU}}\left(\mathbb{C}^{m} \otimes \mathbb{C}^{n}\right)$. Then $\rho=(U \otimes V) \Lambda(U \otimes V)^{\dagger}$ for some $U \in \mathrm{U}(m) / T_{(m)}$ and some $V \in$ $\mathrm{U}(n) / T_{(n)}$. So

$$
\mathrm{d} \rho=(U \otimes V)(\mathrm{d} \Lambda+[\mathrm{d} G, \Lambda])(U \otimes V)^{\dagger} .
$$


Here $\mathrm{d} G=(U \otimes V)^{\dagger} \mathrm{d}(U \otimes V)=\mathrm{d} G_{1} \otimes \mathbb{1}_{n}+\mathbb{1}_{m} \otimes \mathrm{d} G_{2}$, where $\mathrm{d} G_{1}=U^{\dagger} \mathrm{d} U$ and $\mathrm{d} G_{2}=V^{\dagger} \mathrm{d} V$. It suffices to identify the volume element generated by $[\mathrm{d} G, \Lambda]$. We have that

$$
\begin{aligned}
& \langle\mathrm{d} \rho, \mathrm{d} \rho\rangle=\langle\mathrm{d} \Lambda+[\mathrm{d} G, \Lambda], \mathrm{d} \Lambda+[\mathrm{d} G, \Lambda]\rangle \\
& =\langle\mathrm{d} \Lambda, \mathrm{d} \Lambda\rangle+\langle\mathrm{d} \Lambda,[\mathrm{d} G, \Lambda]\rangle+\langle[\mathrm{d} G, \Lambda], \mathrm{d} \Lambda\rangle+\langle[\mathrm{d} G, \Lambda],[\mathrm{d} G, \Lambda]\rangle \\
& =\operatorname{Tr}\left(\mathrm{d} \Lambda^{2}\right)+\operatorname{Tr}\left([\mathrm{d} G, \Lambda]^{+}[\mathrm{d} G, \Lambda]\right),
\end{aligned}
$$

where

$$
\operatorname{Tr}\left([\mathrm{d} G, \Lambda]^{\dagger}[\mathrm{d} G, \Lambda]\right)=2 \operatorname{Tr}(\Lambda \mathrm{d} G \Lambda \mathrm{d} G)-2 \operatorname{Tr}\left(\Lambda^{2} \mathrm{~d} G^{2}\right) .
$$

For $\Lambda=\sum_{i=1}^{m} \sum_{j=1}^{n} \lambda_{i j}|i j\rangle\langle i j|$, we have

$$
\begin{aligned}
& \operatorname{Tr}\left([\mathrm{d} G, \Lambda]^{\dagger}[\mathrm{d} G, \Lambda]\right)=2 \sum_{i, j} \sum_{i^{\prime}, j^{\prime}} \lambda_{i j} \lambda_{i^{\prime} j^{\prime}}\left\langle i j|\mathrm{~d} G| i^{\prime} j^{\prime}\right\rangle\left\langle i^{\prime} j^{\prime}|\mathrm{d} G| i j\right\rangle-2 \sum_{i, j} \lambda_{i j}^{2}\left\langle i j\left|\mathrm{~d} G^{2}\right| i j\right\rangle \\
& =-2 \sum_{i, j} \sum_{i^{\prime}, j^{\prime}} \lambda_{i j} \lambda_{i^{\prime} j^{\prime}}\left|\left\langle i j|\mathrm{~d} G| i^{\prime} j^{\prime}\right\rangle\right|^{2}-2 \sum_{i, j} \lambda_{i j}^{2}\left\langle i j\left|\mathrm{~d} G^{2}\right| i j\right\rangle \\
& =2 \sum_{i, j} \sum_{i^{\prime}, j^{\prime}} \lambda_{i j}^{2}\left|\left\langle i j|\mathrm{~d} G| i^{\prime} j^{\prime}\right\rangle\right|^{2}-2 \sum_{i, j} \sum_{i^{\prime}, j^{\prime}} \lambda_{i j} \lambda_{i^{\prime} j^{\prime}}\left|\left\langle i j|\mathrm{~d} G| i^{\prime} j^{\prime}\right\rangle\right|^{2} \\
& =2 \sum_{i, j} \sum_{i^{\prime}, j^{\prime}} \lambda_{i j}\left(\lambda_{i j}-\lambda_{i^{\prime} j^{\prime}}\right)\left|\left\langle i j|\mathrm{~d} G| i^{\prime} j^{\prime}\right\rangle\right|^{2} .
\end{aligned}
$$

Note that

$$
\begin{aligned}
& \left|\left\langle i j|\mathrm{~d} G| i^{\prime} j^{\prime}\right\rangle\right|^{2}=\left(\left\langle i\left|\mathrm{~d} G_{1}\right| i^{\prime}\right\rangle\left\langle j \mid j^{\prime}\right\rangle+\left\langle j\left|\mathrm{~d} G_{2}\right| j^{\prime}\right\rangle\left\langle i \mid i^{\prime}\right\rangle\right)\left(\overline{\left\langle i\left|\mathrm{~d} G_{1}\right| i^{\prime}\right\rangle\left\langle j \mid j^{\prime}\right\rangle}+\overline{\left\langle j\left|\mathrm{~d} G_{2}\right| j^{\prime}\right\rangle\left\langle i \mid i^{\prime}\right\rangle}\right) \\
& =\left|\left\langle i\left|\mathrm{~d} G_{1}\right| i^{\prime}\right\rangle\right|^{2} \delta_{j j^{\prime}}+\left|\left\langle j\left|\mathrm{~d} G_{2}\right| j^{\prime}\right\rangle\right|^{2} \delta_{i i^{\prime}} \\
& +\left\langle i\left|\mathrm{~d} G_{1}\right| i^{\prime}\right\rangle \overline{\left\langle j\left|\mathrm{~d} G_{2}\right| j^{\prime}\right\rangle} \delta_{i i^{\prime}} \delta_{j j^{\prime}}+\overline{\left\langle i\left|\mathrm{~d} G_{1}\right| i^{\prime}\right\rangle}\left\langle j\left|\mathrm{~d} G_{2}\right| j^{\prime}\right\rangle \delta_{i i^{\prime}} \delta_{j j^{\prime}}
\end{aligned}
$$

Thus,

$$
\begin{aligned}
\operatorname{Tr}\left([\mathrm{d} G, \Lambda]^{\dagger}[\mathrm{d} G, \Lambda]\right)= & 2 \sum_{i, j} \sum_{i^{\prime}, j^{\prime}} \lambda_{i j}\left(\lambda_{i j}-\lambda_{i^{\prime} j^{\prime}}\right)\left|\left\langle i\left|\mathrm{~d} G_{1}\right| i^{\prime}\right\rangle\right|^{2} \delta_{j j^{\prime}} \\
& +2 \sum_{i, j} \sum_{i^{\prime}, j^{\prime}} \lambda_{i j}\left(\lambda_{i j}-\lambda_{i^{\prime} j^{\prime}}\right)\left|\left\langle j\left|\mathrm{~d} G_{2}\right| j^{\prime}\right\rangle\right|^{2} \delta_{i i^{\prime}} \\
& +2 \sum_{i, j} \sum_{i^{\prime}, j^{\prime}} \lambda_{i j}\left(\lambda_{i j}-\lambda_{i^{\prime} j^{\prime}}\right)\left\langle i\left|\mathrm{~d} G_{1}\right| i^{\prime}\right\rangle \overline{\left\langle j\left|\mathrm{~d} G_{2}\right| j^{\prime}\right\rangle} \delta_{i i^{\prime}} \delta_{j j^{\prime}} \\
& +2 \sum_{i, j} \sum_{i^{\prime}, j^{\prime}} \lambda_{i j}\left(\lambda_{i j}-\lambda_{i^{\prime} j^{\prime}}\right) \overline{\left\langle i\left|\mathrm{~d} G_{1}\right| i^{\prime}\right\rangle}\left\langle j\left|\mathrm{~d} G_{2}\right| j^{\prime}\right\rangle \delta_{i i^{\prime}} \delta_{j j^{\prime}} .
\end{aligned}
$$

That is,

$$
\begin{aligned}
\operatorname{Tr}\left([\mathrm{d} G, \Lambda]^{\dagger}[\mathrm{d} G, \Lambda]\right)= & 2 \sum_{i^{\prime}, i, j} \lambda_{i j}\left(\lambda_{i j}-\lambda_{i^{\prime} j}\right)\left|\left\langle i\left|\mathrm{~d} G_{1}\right| i^{\prime}\right\rangle\right|^{2} \\
& +2 \sum_{i, j, j^{\prime}} \lambda_{i j}\left(\lambda_{i j}-\lambda_{i j^{\prime}}\right)\left|\left\langle j\left|\mathrm{~d} G_{2}\right| j^{\prime}\right\rangle\right|^{2}
\end{aligned}
$$


Since $\left|\left\langle i\left|\mathrm{~d} G_{1}\right| i^{\prime}\right\rangle\right|^{2}=\left|\left\langle i^{\prime}\left|\mathrm{d} G_{1}\right| i\right\rangle\right|^{2}$ (both vanish if $i=i^{\prime}$ because $\mathrm{d}_{1}$ is skew-Hermitian), we have

$$
\begin{aligned}
& \sum_{i^{\prime} \neq i}\left[\sum_{j} \lambda_{i j}\left(\lambda_{i j}-\lambda_{i^{\prime} j}\right)\right]\left|\left\langle i\left|\mathrm{~d} G_{1}\right| i^{\prime}\right\rangle\right|^{2} \\
& =\sum_{i<i^{\prime}}\left[\sum_{j} \lambda_{i j}\left(\lambda_{i j}-\lambda_{i^{\prime} j}\right)\right]\left|\left\langle i\left|\mathrm{~d} G_{1}\right| i^{\prime}\right\rangle\right|^{2}+\sum_{i>i^{\prime}}\left[\sum_{j} \lambda_{i j}\left(\lambda_{i j}-\lambda_{i^{\prime} j}\right)\right]\left|\left\langle i\left|\mathrm{~d} G_{1}\right| i^{\prime}\right\rangle\right|^{2} \\
& =\sum_{i<i^{\prime}}\left[\sum_{j} \lambda_{i j}\left(\lambda_{i j}-\lambda_{i^{\prime} j}\right)+\sum_{j} \lambda_{i^{\prime} j}\left(\lambda_{i^{\prime} j}-\lambda_{i j}\right)\right]\left|\left\langle i\left|\mathrm{~d} G_{1}\right| i^{\prime}\right\rangle\right|^{2} \\
& =\sum_{i<i^{\prime}}\left[\sum_{j}\left(\lambda_{i j}-\lambda_{i^{\prime} j}\right)^{2}\right]\left|\left\langle i\left|\mathrm{~d} G_{1}\right| i^{\prime}\right\rangle\right|^{2} .
\end{aligned}
$$

Therefore,

$$
\begin{aligned}
& \langle\mathrm{d} \rho, \mathrm{d} \rho\rangle=\sum_{i, j} \mathrm{~d} \lambda_{i j}^{2}+2 \sum_{i^{\prime} \neq i, j} \lambda_{i j}\left(\lambda_{i j}-\lambda_{i^{\prime} j}\right)\left|\left\langle i\left|\mathrm{~d} G_{1}\right| i^{\prime}\right\rangle\right|^{2}+2 \sum_{i, j \neq j^{\prime}} \lambda_{i j}\left(\lambda_{i j}-\lambda_{i j^{\prime}}\right)\left|\left\langle j\left|\mathrm{~d} G_{2}\right| j^{\prime}\right\rangle\right|^{2} \\
& =\sum_{i, j} \mathrm{~d} \lambda_{i j}^{2}+2 \sum_{i<i^{\prime}}\left(\sum_{j}\left(\lambda_{i j}-\lambda_{i^{\prime} j}\right)^{2}\right)\left|\left\langle i\left|\mathrm{~d} G_{1}\right| i^{\prime}\right\rangle\right|^{2}+2 \sum_{j<j^{\prime}}\left(\sum_{i}\left(\lambda_{i j}-\lambda_{i j^{\prime}}\right)^{2}\right)\left|\left\langle j\left|\mathrm{~d} G_{2}\right| j^{\prime}\right\rangle\right|^{2} .
\end{aligned}
$$

This shows that the Hilbert-Schmidt volume element is given by

$$
\begin{aligned}
& {[\mathrm{d} \rho]=\prod_{i<i^{\prime}} 2\left[\sum_{j}\left(\lambda_{i j}-\lambda_{i^{\prime} j}\right)^{2}\right] \prod_{j<j^{\prime}} 2\left[\sum_{i}\left(\lambda_{i j}-\lambda_{i j^{\prime}}\right)^{2}\right][\mathrm{d} \Lambda]\left[\mathrm{d} G_{1}\right]\left[\mathrm{d} G_{2}\right]} \\
& =2^{\left(\begin{array}{c}
m \\
2
\end{array}\right)+\left(\begin{array}{c}
n \\
2
\end{array}\right)}\left[\prod_{i<i^{\prime}} \sum_{j}\left(\lambda_{i j}-\lambda_{i^{\prime} j}\right)^{2}\right]\left[\prod_{j<j^{\prime}} \sum_{i}\left(\lambda_{i j}-\lambda_{i j^{\prime}}\right)^{2}\right][\mathrm{d} \Lambda]\left[\mathrm{d} G_{1}\right]\left[\mathrm{d} G_{2}\right] .
\end{aligned}
$$

The measure induced by the Lebesgue volume element $\left[\mathrm{d} G_{1}\right]$ on the flag manifold $\mathrm{U}(m) / T_{(m)}$ is the quotient measure. Thus,

$$
\left[\mathrm{dG}_{1}\right]=\frac{\operatorname{vol}_{\mathrm{HS}}(\mathrm{U}(m))}{\operatorname{vol}_{\mathrm{HS}}\left(T_{(m)}\right)} \mathrm{d} \mu_{\mathrm{Haar}}(U),
$$

where $\mathrm{d} \mu_{\text {Haar }}$ denotes the normalized Haar measure. Similarly for $\left[\mathrm{d} G_{2}\right]$. Hence,

$$
[\mathrm{d} \rho]=C_{m, n}\left[\prod_{i<i^{\prime}} \sum_{j}\left(\lambda_{i j}-\lambda_{i^{\prime} j}\right)^{2}\right]\left[\prod_{j<j^{\prime}} \sum_{i}\left(\lambda_{i j}-\lambda_{i j^{\prime}}\right)^{2}\right][\mathrm{d} \Lambda] \mathrm{d} \mu_{\text {Haar }}(U) \mathrm{d} \mu_{\text {Haar }}(V),
$$

where

$$
C_{m, n}=2^{\left(\begin{array}{c}
m \\
2
\end{array}\right)+\left(\begin{array}{c}
n \\
2
\end{array}\right)} \frac{\operatorname{vol}_{\mathrm{HS}}(\mathrm{U}(m))}{\operatorname{vol}_{\mathrm{HS}}\left(T_{(m)}\right)} \frac{\operatorname{vol}_{\mathrm{HS}}(\mathrm{U}(n))}{\operatorname{vol}_{\mathrm{HS}}\left(T_{(n)}\right)}
$$

Integrating over $U$ and $V$ gives the claimed result.

Finally, thanks to Harish-Chandra's volume formula (see Proposition 3.1), the constant $C_{m, n}$ is completely determined by the Lie-algebraic properties of $\mathbf{U}(m)$ and $\mathbf{U}(n)$.

\subsection{Necessary and sufficient conditions for locally diagonalizable two-qubits}

For the most part in this paper, we focus on two-qubits. But first, recall the notion of the Bloch sphere representation for a single qubit. In quantum mechanics, the Bloch sphere is a geometrical representation of 
the pure state space of a two-level quantum mechanical system (qubit). Any qubit state can be represented using the Pauli matrices:

$$
\sigma_{x}=\left(\begin{array}{ll}
0 & 1 \\
1 & 0
\end{array}\right), \quad \sigma_{y}=\left(\begin{array}{cc}
0 & -\mathrm{i} \\
\mathrm{i} & 0
\end{array}\right), \quad \sigma_{z}=\left(\begin{array}{cc}
1 & 0 \\
0 & -1
\end{array}\right) .
$$

More precisely, if we write $\sigma:=\left(\sigma_{x}, \sigma_{y}, \sigma_{z}\right)$, then, for any qubit state $\rho$, we have

$$
\rho=\frac{1}{2}(\mathbb{1}+r(\rho) \cdot \sigma)
$$

for some suitable $r(\rho) \in \mathbb{R}^{3}$, known as the Bloch vector of $\rho$, satisfying $\|\boldsymbol{r}(\rho)\|:=\sqrt{r_{x}^{2}+r_{y}^{2}+r_{z}^{2}} \leqslant 1$. The last term in (2.14) is the usual 'dot product' of 3-tuples.

For reference sake, the commutation and anti-commutation relations satisfied by the Pauli matrices are:

$$
\begin{aligned}
{\left[\sigma_{a}, \sigma_{b}\right] } & :=\sigma_{a} \alpha_{b}-\sigma_{b} \sigma_{a}=2 \epsilon_{a b c} \mathrm{i} \sigma_{c^{\prime}} \\
\left\{\sigma_{a}, \sigma_{b}\right\} & :=\sigma_{a} \sigma_{b}+\sigma_{b} \sigma_{a}=2 \delta_{a b} \mathbb{1},
\end{aligned}
$$

where $\epsilon_{i j k}$ is the Levi-Civita symbol, and $\delta_{i j}$ is the Kronecker delta. The equivariance relation satisfied by the Bloch vector is as follows: For $U \in \mathrm{SU}(2)$, there is some $O \in \mathrm{SO}(3)$ such that

$$
r\left(U \rho U^{\dagger}\right)=\operatorname{Or}(\rho) .
$$

In other words, the adjoint action of a unitary matrix $U \in \mathrm{SU}(2)$ on a qubit state $\rho$ amounts to a rotation of the corresponding Bloch vector of $\rho$. We point out that the correspondence

$$
\begin{array}{ccc}
\mathrm{SU}(2) & \rightarrow \mathrm{SO}(3) \\
U & \mapsto \mathrm{O}
\end{array}
$$

is the universal double covering for $\mathrm{SO}(3)$.

With the Bloch sphere representation (2.14) for a qubit, we have that any two-qubit state can be written in the following way:

$$
\rho_{A B}=\frac{1}{4}\left(\mathbb{1}_{2} \otimes \mathbb{1}_{2}+\boldsymbol{r} \cdot \boldsymbol{\sigma} \otimes \mathbb{1}_{2}+\mathbb{1}_{2} \otimes s \cdot \sigma+\sum_{i, j=x, y, z} t_{i j} \sigma_{i} \otimes \sigma_{j}\right) .
$$

Thus, any two-qubit is given by specifying the 3-dimensional vectors $r$ and $s$ in $\mathbb{R}^{3}$ and the real $3 \times 3$ matrix $T=\left(t_{i j}\right)$.

If two-qubit states $\rho_{A B}$ and $\rho_{A B}^{\prime}$ are $L U$ equivalent, that is, $\rho_{A B}^{\prime}=\left(U_{A} \otimes U_{B}\right) \rho_{A B}\left(U_{A} \otimes U_{B}\right)^{\dagger}$ for some $U_{A}$ and $U_{B}$ in $\mathrm{SU}(2)$, then one can directly check that there are $O_{A}$ and $O_{B}$ in $\mathrm{SO}(3)$ such that

$$
\begin{gathered}
r^{\prime}=O_{A} r, \quad s^{\prime}=O_{B} s, \\
T^{\prime}=O_{A} T O_{B}^{\top} .
\end{gathered}
$$

Conversely, the existence of such $O_{A}$ and $O_{B}$ implies that $\rho_{A B}$ and $\rho_{A B}^{\prime}$ are LU equivalent, thanks to the map (2.17) being a covering. The following theorem from [13] gives another way to check the condition (2.19): 
Theorem 2.2. Two generic two-qubit states are LU equivalent if and only if they have the same values for the following twelve invariants: For $k=0,1,2$,

$$
\begin{aligned}
& \left\langle\boldsymbol{r}\left|\left(T T^{\top}\right)^{k}\right| \boldsymbol{r}\right\rangle, \quad\left\langle\boldsymbol{s}\left|\left(T^{\top} T\right)^{k}\right| \boldsymbol{s}\right\rangle, \\
& \left\langle\boldsymbol{r}\left|\left(T T^{\top}\right)^{k} T\right| \boldsymbol{s}\right\rangle, \quad \operatorname{Tr}\left(\left[T T^{\top}\right]^{k+1}\right) .
\end{aligned}
$$

Our goal of this section is to find an equivalent condition for local diagonalizability of two-qubits. Although it is not absolutely necessary, we will present our argument using the notion of $X$-states, that is, states whose density matrices are of the form

$$
\rho_{X}:=\left(\begin{array}{cccc}
\rho_{11} & 0 & 0 & \rho_{14} \\
0 & \rho_{22} & \rho_{23} & 0 \\
0 & \rho_{32} & \rho_{33} & 0 \\
\rho_{41} & 0 & 0 & \rho_{44}
\end{array}\right) .
$$

In particular, $\rho_{X}$ satisfies the following unit trace and positivity conditions:

(i) $\sum_{j=1}^{4} \rho_{j j}=1$

(ii) $\rho_{22} \rho_{33} \geqslant\left|\rho_{23}\right|^{2}$ and $\rho_{11} \rho_{44} \geqslant\left|\rho_{14}\right|^{2}$

Diagonal states are special cases of $X$-states. We denote by $\mathscr{D}_{X}\left(\mathbb{C}^{2} \otimes \mathbb{C}^{2}\right)$ the set of all two-qubit $X$-states; it is a 7-dimensional submanifold of $\mathrm{D}\left(\mathbb{C}^{2} \otimes \mathbb{C}^{2}\right)$, while $\operatorname{dim}\left(\mathrm{D}\left(\mathbb{C}^{2} \otimes \mathbb{C}^{2}\right)\right)=15$. Thus, owing to Equation (2.8), we have $\operatorname{dim}\left(\mathscr{D}_{X}\left(\mathbb{C}^{2} \otimes \mathbb{C}^{2}\right)\right)=\operatorname{dim}\left(\mathscr{D}_{\mathrm{LU}}\left(\mathbb{C}^{2} \otimes \mathbb{C}^{2}\right)\right)=7$. The Hilbert-Schmidt volume of $\mathscr{D}_{X}\left(\mathbb{C}^{2} \otimes \mathbb{C}^{2}\right)$ has been calculated ${ }^{1}$ in [17]: $\operatorname{vol}_{\mathrm{HS}}\left(\mathscr{D}_{\mathrm{X}}\left(\mathbb{C}^{2} \otimes \mathbb{C}^{2}\right)\right)=\pi^{2} / 630$.

Proposition 2.3. If a two-qubit state is an $X$-state $\rho_{X}$, then it can be written as

$$
\begin{aligned}
\rho_{X}=\frac{1}{4} & \left(\mathbb{1} \otimes \mathbb{1}+a_{z} \sigma_{z} \otimes \mathbb{1}+b_{z} \mathbb{1} \otimes \sigma_{z}+r_{x x} \sigma_{x} \otimes \sigma_{x}\right. \\
& \left.\quad+r_{x y} \sigma_{x} \otimes \sigma_{y}+r_{y x} \sigma_{y} \otimes \sigma_{x}+r_{y y} \sigma_{y} \otimes \sigma_{y}+r_{z z} \sigma_{z} \otimes \sigma_{z}\right),
\end{aligned}
$$

where

$$
\left\{\begin{array} { l } 
{ a _ { z } = \rho _ { 1 1 } - \rho _ { 2 2 } + \rho _ { 3 3 } - \rho _ { 4 4 } , } \\
{ b _ { z } = \rho _ { 1 1 } + \rho _ { 2 2 } - \rho _ { 3 3 } - \rho _ { 4 4 } , } \\
{ r _ { z z } = \rho _ { 1 1 } - \rho _ { 2 2 } - \rho _ { 3 3 } + \rho _ { 4 4 } , }
\end{array} \text { and } \quad \left\{\begin{array}{l}
r_{x x}=\rho_{14}+\rho_{23}+\rho_{32}+\rho_{41}, \\
r_{x y}=\mathrm{i}\left(\rho_{14}+\rho_{23}-\rho_{32}-\rho_{41}\right), \\
r_{y x}=\mathrm{i}\left(\rho_{14}-\rho_{23}+\rho_{32}-\rho_{41}\right), \\
r_{y y}=-\rho_{14}+\rho_{23}+\rho_{32}-\rho_{41} .
\end{array}\right.\right.
$$

Moreover, all eigenvalues of $\rho_{X}$ are given by

$$
\begin{aligned}
& \lambda_{1,2}\left(\rho_{X}\right)=\frac{1}{4}\left(\left(1+r_{z z}\right) \pm \sqrt{\left(a_{z}+b_{z}\right)^{2}+\left(r_{x x}-r_{y y}\right)^{2}+\left(r_{x y}+r_{y x}\right)^{2}}\right), \\
& \lambda_{3,4}\left(\rho_{X}\right)=\frac{1}{4}\left(\left(1-r_{z z}\right) \pm \sqrt{\left(a_{z}-b_{z}\right)^{2}+\left(r_{x x}+r_{y y}\right)^{2}+\left(r_{x y}-r_{y x}\right)^{2}}\right) .
\end{aligned}
$$

\footnotetext{
${ }^{1}$ The Hilbert-Schmidt volume stated here is a correction to the result of Milz and Strunz [17]; they misused a factor leading to an incorrect Hilbert-Schmidt volume of $\pi^{2} / 5040$. Their calculation of the Euclid volume of $X$-states is correct.
} 
Applying Proposition 2.3 to a $4 \times 4$ diagonal state $\Lambda=\operatorname{diag}\left(\lambda_{1}, \lambda_{2}, \lambda_{3}, \lambda_{4}\right)$, we have that

$$
\Lambda=\frac{1}{4}\left(\mathbb{1}_{2} \otimes \mathbb{1}_{2}+a_{z} \sigma_{z} \otimes \mathbb{1}_{2}+b_{z} \mathbb{1}_{2} \otimes \sigma_{z}+r_{z z} \sigma_{z} \otimes \sigma_{z}\right),
$$

where

$$
\left\{\begin{array}{l}
a_{z}=\lambda_{1}-\lambda_{2}+\lambda_{3}-\lambda_{4} \\
b_{z}=\lambda_{1}+\lambda_{2}-\lambda_{3}-\lambda_{4} \\
r_{z z}=\lambda_{1}-\lambda_{2}-\lambda_{3}+\lambda_{4}
\end{array} .\right.
$$

Then $r(\Lambda)=a_{z}|3\rangle, s(\Lambda)=b_{z}|3\rangle$, and $T(\Lambda)=r_{z z}|3\rangle\langle 3|$, where $|3\rangle:=(0,0,1)^{\top}$. Applying Theorem 2.2 to a state $\rho$ that is LU equivalent to the diagonal form $\Lambda$ gives us the following result:

Theorem 2.4. A generic two-qubit state $\rho$ is locally diagonalizable if and only if the following conditions are satisfied:

$$
\boldsymbol{r}=a_{z} O_{A}|3\rangle, \quad s=b_{z} O_{B}|3\rangle, \quad T=r_{z z} O_{A}|3\rangle\langle 3| O_{B}^{\top}
$$

where $O_{A}$ and $O_{B}$ are in $\mathrm{SO}(3)$, and the triple $\left(a_{z}, b_{z}, r_{z z}\right)$ is given by (2.27).

The above theorem characterizes locally diagonalizable two-qubit states. Its generalization to higher dimensions is apparently unknown at present.

\subsection{The Hilbert-Schmidt volume of locally diagonalizable two-qubits}

The primary goal of this section is to compute the HS volume of locally diagonalizable two-qubits. The result is the following theorem:

Theorem 2.5. The Hilbert-Schmidt volume of $\mathscr{D}_{\mathrm{LU}}\left(\mathbb{C}^{2} \otimes \mathbb{C}^{2}\right)$ is given by

$$
\operatorname{vol}_{\mathrm{HS}}\left(\mathscr{D}_{\mathrm{LU}}\left(\mathbb{C}^{2} \otimes \mathbb{C}^{2}\right)\right)=\frac{(4 \pi)^{2}}{105}
$$

Proof. If $\rho \in \mathscr{D}_{\mathrm{LU}}\left(\mathbb{C}^{2} \otimes \mathbb{C}^{2}\right)$, then

$$
\rho=(U \otimes V) \Lambda(U \otimes V)^{\dagger}
$$

where $U$ and $V$ are in $U(2)$, and $\Lambda=\operatorname{diag}\left(\lambda_{1}, \lambda_{2}, \lambda_{3}, \lambda_{4}\right)$ with $\lambda_{j}$ 's being pairwise different and satisfying $\sum_{j} \lambda_{j}=1$. In the proof of Lemma 2.1, we see from (2.11) that

$$
[\mathrm{d} \rho]=C_{2,2}\left[\left(\lambda_{1}-\lambda_{2}\right)^{2}+\left(\lambda_{3}-\lambda_{4}\right)^{2}\right]\left[\left(\lambda_{1}-\lambda_{3}\right)^{2}+\left(\lambda_{2}-\lambda_{4}\right)^{2}\right][\mathrm{d} \Lambda] \mathrm{d} \mu_{\text {Haar }}(U) \mathrm{d} \mu_{\text {Haar }}(V) .
$$

Then,

$$
\begin{aligned}
\delta(1-\operatorname{Tr}(\rho))[\mathrm{d} \rho]= & C_{2,2} \delta\left(1-\sum_{j} \lambda_{j}\right) \\
& \times\left[\left(\lambda_{1}-\lambda_{2}\right)^{2}+\left(\lambda_{3}-\lambda_{4}\right)^{2}\right]\left[\left(\lambda_{1}-\lambda_{3}\right)^{2}+\left(\lambda_{2}-\lambda_{4}\right)^{2}\right][\mathrm{d} \Lambda]
\end{aligned}
$$


Thus

$$
\begin{aligned}
& \int_{\mathscr{D}_{\mathrm{LU}}\left(\mathbb{C}^{2} \otimes \mathbb{C}^{2}\right)} \delta(1-\operatorname{Tr}(\rho))[\mathrm{d} \rho] \\
& =C_{2,2} \int \delta\left(1-\sum_{j} \lambda_{j}\right)\left[\left(\lambda_{1}-\lambda_{2}\right)^{2}+\left(\lambda_{3}-\lambda_{4}\right)^{2}\right]\left[\left(\lambda_{1}-\lambda_{3}\right)^{2}+\left(\lambda_{2}-\lambda_{4}\right)^{2}\right][\mathrm{d} \Lambda] .
\end{aligned}
$$

It is known that (for details, see [20])

$$
\operatorname{vol}_{\mathrm{HS}}(\mathrm{U}(k))=\frac{(2 \pi)^{\left(\begin{array}{c}
k+1 \\
2
\end{array}\right)}}{\prod_{j=1}^{k} \Gamma(j)} .
$$

So $\operatorname{vol}_{\mathrm{HS}}(\mathrm{U}(2))=(2 \pi)^{3}$ and $\operatorname{vol}_{\mathrm{HS}}\left(T_{(2)}\right)=\operatorname{vol}_{\mathrm{HS}}\left(\mathrm{U}(1)^{2}\right)=\left(\operatorname{vol}_{\mathrm{HS}}(\mathrm{U}(1))^{2}=(2 \pi)^{2}\right.$. Therefore,

$$
C_{2,2}=(4 \pi)^{2}
$$

and we have

$$
\begin{aligned}
& \operatorname{vol}_{\mathrm{HS}}\left(\mathscr{D}_{\mathrm{LU}}\left(\mathbb{C}^{2} \otimes \mathbb{C}^{2}\right)\right)=\int_{\mathscr{D}_{\mathrm{LU}}\left(\mathbb{C}^{2} \otimes \mathbb{C}^{2}\right)} \delta(1-\operatorname{Tr}(\rho))[\mathrm{d} \rho] \\
& =(4 \pi)^{2} \int \delta\left(1-\sum_{j} \lambda_{j}\right)\left[\left(\lambda_{1}-\lambda_{2}\right)^{2}+\left(\lambda_{3}-\lambda_{4}\right)^{2}\right]\left[\left(\lambda_{1}-\lambda_{3}\right)^{2}+\left(\lambda_{2}-\lambda_{4}\right)^{2}\right] \prod_{j=1}^{4} \mathrm{~d} \lambda_{j} .
\end{aligned}
$$

It remains to evaluate the last integral. Note that

$$
\begin{aligned}
& {\left[\left(\lambda_{1}-\lambda_{2}\right)^{2}+\left(\lambda_{3}-\lambda_{4}\right)^{2}\right]\left[\left(\lambda_{1}-\lambda_{3}\right)^{2}+\left(\lambda_{2}-\lambda_{4}\right)^{2}\right]} \\
& =\left(\lambda_{1}-\lambda_{2}\right)^{2}\left(\lambda_{1}-\lambda_{3}\right)^{2}+\left(\lambda_{3}-\lambda_{1}\right)^{2}\left(\lambda_{3}-\lambda_{4}\right)^{2}+\left(\lambda_{2}-\lambda_{1}\right)^{2}\left(\lambda_{2}-\lambda_{4}\right)^{2}+\left(\lambda_{4}-\lambda_{2}\right)^{2}\left(\lambda_{4}-\lambda_{3}\right)^{2} .
\end{aligned}
$$

The following four integrals are equal:

$$
\begin{aligned}
& \int \delta\left(1-\sum_{j} \lambda_{j}\right)\left(\lambda_{1}-\lambda_{2}\right)^{2}\left(\lambda_{1}-\lambda_{3}\right)^{2} \prod_{j=1}^{4} \mathrm{~d} \lambda_{j} \\
& \int \delta\left(1-\sum_{j} \lambda_{j}\right)\left(\lambda_{3}-\lambda_{1}\right)^{2}\left(\lambda_{3}-\lambda_{4}\right)^{2} \prod_{j=1}^{4} \mathrm{~d} \lambda_{j} \\
& \int \delta\left(1-\sum_{j} \lambda_{j}\right)\left(\lambda_{2}-\lambda_{1}\right)^{2}\left(\lambda_{2}-\lambda_{4}\right)^{2} \prod_{j=1}^{4} \mathrm{~d} \lambda_{j} \\
& \int \delta\left(1-\sum_{j} \lambda_{j}\right)\left(\lambda_{4}-\lambda_{2}\right)^{2}\left(\lambda_{4}-\lambda_{3}\right)^{2} \prod_{j=1}^{4} \mathrm{~d} \lambda_{j} .
\end{aligned}
$$

Let

$$
f(t)=\int \delta\left(t-\sum_{j} \lambda_{j}\right)\left(\lambda_{1}-\lambda_{2}\right)^{2}\left(\lambda_{1}-\lambda_{3}\right)^{2} \prod_{j=1}^{4} \mathrm{~d} \lambda_{j} .
$$


Performing the Laplace transformation $(t \rightarrow s)$ on $f(t)$, we get, for $s>0$,

$$
\begin{aligned}
\widetilde{f}(s) & =\mathscr{L}(f)(s)=\int_{0}^{\infty} f(t) e^{-s t} \mathrm{~d} t \\
& =\int_{0}^{\infty} \int_{0}^{\infty} \int_{0}^{\infty} \int_{0}^{\infty}\left[\int_{0}^{\infty} \mathrm{d} t e^{-s t} \delta\left(t-\sum_{j} \lambda_{j}\right)\right]\left(\lambda_{1}-\lambda_{2}\right)^{2}\left(\lambda_{1}-\lambda_{3}\right)^{2} \prod_{j=1}^{4} \mathrm{~d} \lambda_{j} . \\
& =\int_{0}^{\infty} \int_{0}^{\infty} \int_{0}^{\infty} \int_{0}^{\infty} \exp \left(-s \sum_{j} \lambda_{j}\right)\left(\lambda_{1}-\lambda_{2}\right)^{2}\left(\lambda_{1}-\lambda_{3}\right)^{2} \prod_{j=1}^{4} \mathrm{~d} \lambda_{j} .
\end{aligned}
$$

By change of variables,

$$
\begin{aligned}
\tilde{f}(s) & =s^{-8} \int_{0}^{\infty} \int_{0}^{\infty} \int_{0}^{\infty} \int_{0}^{\infty} \exp \left(-\sum_{j=1}^{4} x_{j}\right)\left(x_{1}-x_{2}\right)^{2}\left(x_{1}-x_{3}\right)^{2} \prod_{j=1}^{4} \mathrm{~d} x_{j} \\
& =s^{-8} \int_{0}^{\infty} \int_{0}^{\infty} \int_{0}^{\infty} \exp \left(-\sum_{j=1}^{3} x_{j}\right)\left(x_{1}-x_{2}\right)^{2}\left(x_{1}-x_{3}\right)^{2} \prod_{j=1}^{3} \mathrm{~d} x_{j} .
\end{aligned}
$$

Since

$$
\left(x_{1}-x_{2}\right)^{2}\left(x_{1}-x_{3}\right)^{2}=x_{1}^{4}-2\left(x_{2}+x_{3}\right) x_{1}^{3}+\left(x_{2}^{2}+x_{3}^{2}+4 x_{2} x_{3}\right) x_{1}^{2}-2 x_{2} x_{3}\left(x_{2}+x_{3}\right) x_{1}+x_{2}^{2} x_{3}^{2}
$$

we have

$$
\begin{aligned}
& \int_{0}^{\infty} \mathrm{d} x_{1} e^{-x_{1}}\left(x_{1}-x_{2}\right)^{2}\left(x_{1}-x_{3}\right)^{2} \\
& =\Gamma(5)-2\left(x_{2}+x_{3}\right) \Gamma(4)+\left(x_{2}^{2}+x_{3}^{2}+4 x_{2} x_{3}\right) \Gamma(3)-2 x_{2} x_{3}\left(x_{2}+x_{3}\right) \Gamma(2)+x_{2}^{2} x_{3}^{2},
\end{aligned}
$$

where we utilized the integral representation of the Gamma function $\Gamma(z)=\int_{0}^{\infty} x^{z-1} e^{-x} \mathrm{~d} x$. Hence,

$$
\begin{aligned}
& \int_{0}^{\infty} \int_{0}^{\infty} \int_{0}^{\infty} \mathrm{d} x_{1} \mathrm{~d} x_{2} \mathrm{~d} x_{3} e^{-x_{1}} e^{-x_{2}} e^{-x_{3}}\left(x_{1}-x_{2}\right)^{2}\left(x_{1}-x_{3}\right)^{2} \\
& =\Gamma(5)-2[\Gamma(2)+\Gamma(2)] \Gamma(4)+[\Gamma(3)+\Gamma(3)+4 \Gamma(2) \Gamma(2)] \Gamma(3) \\
& \quad-2[\Gamma(3) \Gamma(2)+\Gamma(2) \Gamma(3)] \Gamma(2)+\Gamma(3) \Gamma(3) \\
& =12 .
\end{aligned}
$$

Therefore,

$$
\widetilde{f}(s)=12 \cdot s^{-8} \text {. }
$$

Then,

$$
f(t)=\mathscr{L}^{-1}(\widetilde{f})(t)=12 \cdot \frac{t^{7}}{7 !}=\frac{1}{420} t^{7}
$$

Finally,

$$
\operatorname{vol}_{\mathrm{HS}}\left(\mathscr{D}_{\mathrm{LU}}\left(\mathbb{C}^{2} \otimes \mathbb{C}^{2}\right)\right)=(4 \pi)^{2} \cdot 4 f(1)=\frac{(4 \pi)^{2}}{105} .
$$

This completes the proof. 
Remark 2.6. The evaluation of $f(1)$ from (2.35) could have been done using the formulas for the density of Dirichlet distributions; the Dirichlet distribution of order $N$ with parameters $\alpha_{j}>0(j=1, \ldots, N)$ has a probability density function relative to the Lebesgue measure on $\mathbb{R}^{N-1}$ given by

$$
p\left(x_{1}, \ldots, x_{N} ; \alpha_{1}, \ldots, \alpha_{N}\right):=C\left(\alpha_{1}, \ldots, \alpha_{N}\right) \delta\left(1-\sum_{k=1}^{N} x_{k}\right) \prod_{k=1}^{N} x_{k}^{\alpha_{k}-1},
$$

where the normalization constant $C\left(\alpha_{1}, \ldots, \alpha_{N}\right)$ is given by

$$
C\left(\alpha_{1}, \ldots, \alpha_{N}\right)=\frac{\Gamma\left(\sum_{k=1}^{N} \alpha_{k}\right)}{\prod_{k=1}^{N} \Gamma\left(\alpha_{k}\right)} .
$$

Here $\Gamma$ denotes the Gamma function as usual.

Remark 2.7. Seeking to generalize the volume formula, we can attempt to apply the argument used in the proof of Theorem 2.5 to arbitrary bipartite states. That would lead us to:

$$
\begin{aligned}
& \operatorname{vol}_{\mathrm{HS}}\left(\mathscr{D}_{\mathrm{LU}}\left(\mathbb{C}^{m} \otimes \mathbb{C}^{n}\right)\right)=\int_{\mathscr{D}_{\mathrm{LU}}\left(\mathbb{C}^{m} \otimes \mathbb{C}^{n}\right)} \delta(1-\operatorname{Tr}(\rho))[\mathrm{d} \rho] \\
& =C_{m, n} \int \delta\left(1-\sum_{i=1}^{m} \sum_{j=1}^{n} \lambda_{i j}\right) \\
& \quad \times\left(\prod_{1 \leqslant i<i^{\prime} \leqslant m} \sum_{j=1}^{n}\left(\lambda_{i j}-\lambda_{i^{\prime} j}\right)^{2}\right)\left(\prod_{1 \leqslant j<j^{\prime} \leqslant n} \sum_{i=1}^{m}\left(\lambda_{i j}-\lambda_{i j^{\prime}}\right)^{2}\right) \prod_{i=1}^{m} \prod_{j=1}^{n} \mathrm{~d} \lambda_{i j} .
\end{aligned}
$$

Here the constant $C_{m, n}$ is from (2.12). The above integral can in principle be evaluated for a given pair $(m, n)$, giving us a volume formula. But carrying out the computation seems complicated (we tried to use computers for the qubit-qutrit case $(m, n)=(2,3)$ without success). We certainly do not have a unified closed expression for the integral (2.45). We can still try to obtain some insight. Let

$$
P\left(\lambda_{i j}\right):=\left(\prod_{1 \leqslant i<i^{\prime} \leqslant m} \sum_{j=1}^{n}\left(\lambda_{i j}-\lambda_{i^{\prime} j}\right)^{2}\right)\left(\prod_{1 \leqslant j<j^{\prime} \leqslant n} \sum_{i=1}^{m}\left(\lambda_{i j}-\lambda_{i j^{\prime}}\right)^{2}\right) .
$$

This is a homogeneous multivariate polynomial of $m n$ variables $\lambda_{i j}$ with integer coefficients. Upon expansion, we get

$$
P\left(\lambda_{i j}\right)=\sum \prod_{i, j} \lambda_{i j}^{\gamma_{i j}-1}
$$

where $\gamma_{i j}$ 's are positive integers, and the summation is finite. From Remark 2.6, we can infer that the HS volume of all locally diagonalizable bipartite states is always a power of $\pi$ times a rational number.

\section{Harish-Chandra's volume formula}

Let $\mathbf{U}(m)$ be the unitary group acting on $\mathbb{C}^{m}$ with Lie algebra $\mathfrak{u}(m)$. Denote by $T_{(m)}$ the standard maximal torus of $\mathbf{U}(m)$, namely, the set of diagonal matrices in $\mathbf{U}(m)$. Note that $T_{(n)} \cong \mathbf{U}(1)^{\times n}$ and that the Lie algebra $\mathfrak{t}_{(m)}$ of $T_{(m)}$ is isomorphic to $\sqrt{-1} \mathbb{R}^{m}$. Without loss of generality, we take $\mathfrak{t}_{(m)}$ as the set of diagonal matrices with purely imaginary diagonal entries. Let $K=\mathrm{U}(m) \otimes \mathrm{U}(n)$. Then for the Lie algebra $\mathfrak{k}$ of $K$ we have

$$
\mathfrak{k}=\mathfrak{u}(m) \otimes \mathbb{1}_{n}+\mathbb{1}_{m} \otimes \mathfrak{u}(n) .
$$


Similarly, for the Lie algebra $\mathfrak{t}$ of the maximal torus $T=T_{(m)} \otimes T_{(n)}$ of $K$, we have

$$
\mathfrak{t}=\mathfrak{t}_{(m)} \otimes \mathbb{1}_{n}+\mathbb{1}_{m} \otimes \mathfrak{t}_{(n)} .
$$

It is a routine exercise to see that $\operatorname{dim}(\mathfrak{t})=\operatorname{dim}\left(\mathfrak{t}_{(m)}\right)+\operatorname{dim}\left(\mathfrak{t}_{(n)}\right)-1=m+n-1$. We shall see that (Proposition 3.4) $\operatorname{dim}(\mathfrak{k})=\operatorname{dim}(\mathfrak{u}(m))+\operatorname{dim}(\mathfrak{u}(n))-1=m^{2}+n^{2}-1$. We also have

$$
[\mathrm{U}(m) \otimes \mathbf{U}(n)] /\left[T_{(m)} \otimes T_{(n)}\right] \simeq\left[\mathrm{U}(m) / T_{(m)}\right] \otimes\left[\mathrm{U}(n) / T_{(n)}\right] .
$$

It goes without saying that, for volumes of quotient spaces, we shall use quotient measures. For details on quotient measures, we refer to [8, Sec. 3.13].

Proposition 3.1 (Harish-Chandra's volume formula [10]). Let $K$ be a compact, connected Lie group. Let $T$ be the maximal torus of $K$. Endow $K$ with the metric $g$ induced by an invariant inner product on the Lie algebra $\mathfrak{k}$ of $K$; endow $T$ with the subspace metric. Then the Riemannian volumes of $K$ and $T$ satisfy

$$
\frac{\operatorname{vol}_{g}(K)}{\operatorname{vol}_{g}(T)}=\prod_{\alpha \in \Phi_{\mathfrak{k}}^{+}} \frac{2 \pi}{\langle\alpha, \omega\rangle},
$$

where $\Phi_{\mathfrak{k}}^{+}$is the set of all positive roots for $\mathfrak{k}$ and $\omega:=\frac{1}{2} \sum_{\alpha \in \Phi_{\mathfrak{k}}^{+}} \alpha$, which is Weyl vector, i.e., the half the sum of all positive roots of $\mathfrak{k}$.

Remark 3.2. If we endow the flag manifold $K / T$ with the quotient measure, then the volume ratio (3.4) equals the volume of $K / T$. The beauty of Harish-Chandra's formula is that the volume ratio is completely determined by the Lie-algebraic properties of $\mathfrak{k}$ and is independent of the choice of the invariant metric for $K$ and T. For modern expositions on Harish-Chandra's formula, we refer to [5, Cor. 7.27] or [8, Eq. 3.14.13].

\subsection{Hilbert-Schmidt volume}

Our focus is on the Hilbert-Schmidt measure on $K$ and $T$. We shall denote the volume of $K / T$ relative to the quotient measure as $\operatorname{vol}_{\mathrm{HS}}(K / T)$. Hence,

$$
\operatorname{vol}_{\mathrm{HS}}(K / T)=\frac{\operatorname{vol}_{\mathrm{HS}}(K)}{\operatorname{vol}_{\mathrm{HS}}(T)} .
$$

Example 3.3. Let us calculate the volume of the flag manifold $\mathrm{U}(n) / T_{(n)}$. The Lie algebra $\mathfrak{t}_{(n)}$ is the set of all diagonal matrices in $\mathfrak{u}(n)$. We can take the following set as the set of all positive roots of $\mathfrak{u}(n)$ :

$$
\Phi_{\mathfrak{u}(n)}^{+}=\left\{\alpha_{i j} \in \mathfrak{t}_{(n)}^{*}: \alpha_{i j}(X)=x_{i}-x_{j} \text { for any } X=\operatorname{diag}\left(x_{1}, \ldots, x_{n}\right), i<j\right\} .
$$

We can view a diagonal matrix $X$ as a real vector $X=\left(x_{1}, \ldots, x_{n}\right)$. In turn, we may view $\alpha_{i j}$ as the real vector

$$
\alpha_{i j}=(\cdots \overbrace{1}^{i} \cdots \overbrace{-1}^{j} \cdots) \quad(1 \leqslant i<j \leqslant n),
$$

where $\cdot$ stands for zeroes. Then $\alpha_{i j}(X)=\left\langle\alpha_{i j}, X\right\rangle$, where the right-hand side denotes the dot product of $\alpha_{i j}$ and $X$. The Weyl vector is

$$
\omega=\frac{1}{2} \sum_{i<j} \alpha_{i j}=\frac{1}{2}(n-1, n-3, \ldots, 3-n, 1-n) .
$$


Let $\left\{e_{j}: j=1, \ldots, n\right\}$ be the standard orthonormal basis for $\mathbb{R}^{n}$. Then $\alpha_{i j}=e_{i}-e_{j}$ and $\omega=\sum_{j}\left(\frac{n+1}{2}-j\right) e_{j}$. So $\left\langle\alpha_{i j}, \omega\right\rangle=\omega_{i}-\omega_{j}=\left(\frac{n+1}{2}-i\right)-\left(\frac{n+1}{2}-j\right)=j-i$. Then, by (3.4),

$$
\operatorname{vol}_{\mathrm{HS}}\left(\mathrm{U}(n) / T_{(n)}\right)=\frac{\operatorname{vol}_{\mathrm{HS}}(\mathrm{U}(n))}{\operatorname{vol}_{\mathrm{HS}}\left(T_{(n)}\right)}=\prod_{i<j} \frac{2 \pi}{\left\langle\alpha_{i j}, \boldsymbol{\omega}\right\rangle}=\prod_{i<j} \frac{2 \pi}{j-i}=\frac{(2 \pi)^{\left(\begin{array}{c}
n \\
2
\end{array}\right)}}{\prod_{j=1}^{n} \Gamma(j)} .
$$

A direct computation yields (see, for instance, [20])

$$
\operatorname{vol}_{\mathrm{HS}}\left(T_{(n)}\right)=(2 \pi)^{n} \text {. }
$$

Then

$$
\operatorname{vol}_{\mathrm{HS}}(\mathrm{U}(n))=\frac{(2 \pi)^{\frac{n(n+1)}{2}}}{\prod_{j=1}^{n} \Gamma(j)} .
$$

We now move on to describing the roots of $\mathfrak{k}$. Let $\Phi_{\mathfrak{u}(m)}$ denote the set of roots of a Lie algebra $\mathfrak{u}(m)$. For each $\alpha^{(m)} \in \Phi_{\mathfrak{u}(m)}$, denote its associated root space by $\mathfrak{u}(m)_{\alpha^{(m)}}$. Let $\tau_{m}(\cdot):=\frac{1}{m} \operatorname{Tr}(\cdot)$ be the normalized trace form on the $m \times m$ matrices so that $\tau_{m}\left(\mathbb{1}_{m}\right)=1$. For any $\alpha^{(m)} \in \Phi_{\mathfrak{u}(m)}$, the tensor product $\alpha^{(m)} \otimes \tau_{n}$ is a purely imaginary-valued $\mathbb{R}$-linear map $\mathfrak{t}_{(m)} \otimes \mathbb{1}_{n} \rightarrow \mathbb{C}$. Extend the domain of $\alpha^{(m)} \otimes \tau_{n}$ by zero to $\mathfrak{t}=\mathfrak{t}_{(m)} \otimes \mathbb{1}_{n}+\mathbb{1}_{m} \otimes \mathfrak{t}_{(n)}$ (recall that the intersection $\left(\mathfrak{t}_{(m)} \otimes \mathbb{1}_{n}\right) \cap\left(\mathbb{1}_{m} \otimes \mathfrak{t}_{(n)}\right)=\sqrt{-1} \mathbb{R} \cdot \mathbb{1}_{m} \otimes \mathbb{1}_{n}$, and on such intersection, $\alpha^{(m)} \otimes \tau_{n}$ yields the value 0 because $\alpha^{(m)}$ depends only on the differences in the diagonal entries). We denote this extension by $\tilde{\alpha}^{(m)}=\alpha^{(m)} \otimes \tau_{n}$. Symmetrically, for each root $\alpha^{(n)}$ of $\mathfrak{u}(n)$, we denote by $\tilde{\alpha}^{(n)}$ the purely imaginary-valued $\mathbb{R}$-linear map $\mathfrak{t} \rightarrow \mathbb{C}$ obtained by extending $\tau_{m} \otimes \alpha^{(n)}$ by zero.

Proposition 3.4. Let $\widetilde{\Phi}_{\mathfrak{u}(m)}=\left\{\tilde{\alpha}^{(m)} \mid \alpha^{(m)} \in \Phi_{\mathfrak{u}(m)}\right\}$ and $\widetilde{\Phi}_{\mathfrak{u}(n)}=\left\{\tilde{\alpha}^{(n)} \mid \alpha^{(n)} \in \Phi_{\mathfrak{u}(n)}\right\}$. Their disjoint union yields the set of roots for $\mathfrak{k}=\mathfrak{u}(m) \otimes \mathbb{1}_{n}+\mathbb{1}_{m} \otimes \mathfrak{u}(n)$ :

$$
\Phi_{\mathfrak{k}}=\widetilde{\Phi}_{\mathfrak{u}(m)} \bigsqcup \widetilde{\Phi}_{\mathfrak{u}(n)} .
$$

The root space associated with $\tilde{\alpha}^{(m)} \in \widetilde{\Phi}_{\mathfrak{u}(m)}$ is $\tilde{\mathfrak{u}}(m):=\mathfrak{u}(m) \otimes \mathbb{1}_{n}$. The root space associated with $\tilde{\alpha}^{(n)} \in \widetilde{\Phi}_{\mathfrak{u}(n)}$ is $\tilde{\mathfrak{u}}(n):=\mathbb{1}_{m} \otimes \mathfrak{u}(n)$.

Proof. Take the root space decompositions (the subscript $\mathbb{C}$ denotes complexification)

$$
\begin{aligned}
\mathfrak{u}(m)_{\mathbf{C}} & =\mathfrak{t}_{(m), \mathbb{C}} \oplus\left(\bigoplus_{i \neq j}^{m} \mathfrak{u}(m)_{\alpha_{i j}^{(m)}}\right), \\
\mathfrak{u}(n)_{\mathbf{C}} & =\mathfrak{t}_{(n), \mathbb{C}} \oplus\left(\bigoplus_{k \neq l}^{n} \mathfrak{u}(n)_{\alpha_{k l}^{(n)}}\right) .
\end{aligned}
$$

Then,

$$
\begin{aligned}
& \mathfrak{k}_{\mathbb{C}}=\mathfrak{u}(m)_{\mathbb{C}} \otimes \mathbb{1}_{n}+\mathbb{1}_{m} \otimes \mathfrak{u}(n)_{\mathbb{C}} \\
& =\left(\mathfrak{t}_{(m), \mathbb{C}} \otimes \mathbb{1}_{n}+\mathbb{1}_{m} \otimes \mathfrak{t}_{(n), \mathbb{C}}\right)+\left(\bigoplus_{i \neq j}^{m} \mathfrak{u}(m)_{\alpha_{i j}^{(m)}} \otimes \mathbb{1}_{n}\right)+\left(\bigoplus_{k \neq l}^{n} \mathbb{1}_{m} \otimes \mathfrak{u}(n)_{\alpha_{k l}^{(n)}}\right) .
\end{aligned}
$$

We need to show that $\mathfrak{u}(m)_{\alpha_{i j}^{(m)}} \otimes \mathbb{1}_{n}$ and $\mathbb{1}_{m} \otimes \mathfrak{u}(n)_{\alpha_{k l}^{(n)}}$ are root spaces for $\mathfrak{k}$. Take an arbitrary vector $Z \in \mathfrak{t}_{(m), \mathbb{C}} \otimes \mathbb{1}_{n}+\mathbb{1}_{m} \otimes \mathfrak{t}_{(n), \mathbb{C}} ;$ then

$$
Z=X \otimes \mathbb{1}_{n}+\mathbb{1}_{m} \otimes Y
$$


for some $X \in \mathfrak{u}(m)_{\mathbb{C}}$ and $Y \in \mathfrak{u}(n)_{\mathbb{C}}$. Observe that

$$
\left[Z, \mathfrak{u}(m)_{\alpha_{i j}^{(m)}} \otimes \mathbb{1}_{n}\right]=\left[X, \mathfrak{u}(m)_{\alpha_{i j}^{(m)}}\right] \otimes \mathbb{1}_{n}=\alpha_{i j}^{(m)}(X)\left(\mathfrak{u}(m)_{\alpha_{i j}^{(m)}} \otimes \mathbb{1}_{n}\right) .
$$

So $\mathfrak{u}(m)_{\alpha_{i j}^{(m)}} \otimes \mathbb{1}_{n}$ is indeed a root space for $\mathfrak{k}$. Similar argument proves that $\mathbb{1}_{m} \otimes \mathfrak{u}(n)_{\alpha_{k l}^{(n)}}$ is a root space for $\mathfrak{k}$.

We have demonstrated so far that $\Phi_{\mathfrak{k}}=\widetilde{\Phi}_{\mathfrak{u}(m)} \cup \widetilde{\Phi}_{\mathfrak{u}(n)}$. To show that this is a disjoint union, we claim that, for any pair $\left(\alpha^{(m)}, \alpha^{(n)}\right) \in \Phi_{\mathfrak{u}(m)} \times \Phi_{\mathfrak{u}(n)}$, the two root spaces $\mathfrak{u}(m)_{\alpha(m)} \otimes \mathbb{1}_{n}$ and $\mathbb{1}_{m} \otimes \mathfrak{u}(n)_{\alpha^{(n)}}$ are distinct. Since root spaces are 1-dimensional, our claim is equivalent to saying that

$$
\left(\mathfrak{u}(m)_{\alpha^{(m)}} \otimes \mathbb{1}_{n}\right) \cap\left(\mathbb{1}_{m} \otimes \mathfrak{u}(n)_{\alpha^{(n)}}\right)=\{0\} .
$$

To prove the above equation, say $X \in \mathfrak{u}(m)_{\alpha^{(m)}}$ and $Y \in \mathfrak{u}(n)_{\alpha^{(n)}}$. As root vectors, the nonzero entries of $X$ and $Y$ are all off-diagonal. Now suppose $X \otimes \mathbb{1}_{n}=\mathbb{1}_{m} \otimes Y$. Because all diagonal entries of both $X$ and $Y$ are zero, then it is easy to see that both $X$ and $Y$ are zero matrices. This completes the proof.

With the above Proposition 3.4 at hand, we can calculate $\operatorname{vol}_{\mathrm{HS}}(K)$ for $K=\mathrm{U}(m) \otimes \mathrm{U}(n)$ using HarishChandra's formula:

$$
\begin{aligned}
& \operatorname{vol}_{\mathrm{HS}}(\mathrm{U}(m) \otimes \mathrm{U}(n))=\operatorname{vol}_{\mathrm{HS}}\left(T_{(m)} \otimes T_{(n)}\right) \prod_{\alpha \in \Phi_{\mathfrak{k}}^{+}} \frac{2 \pi}{\langle\alpha, \omega\rangle} \\
& =(2 \pi)^{m+n-1} \prod_{\alpha^{(m)} \in \Phi_{\mathfrak{u}(m)}^{+}} \frac{2 \pi}{\left\langle\tilde{\alpha}^{(m)}, \widetilde{\omega}_{\mathfrak{u}(m)}+\widetilde{\omega}_{\mathfrak{u}(n)}\right\rangle} \prod_{\alpha^{(n)} \in \Phi_{\mathfrak{u}(n)}^{+}} \frac{2 \pi}{\left\langle\tilde{\alpha}^{(n)}, \widetilde{\omega}_{\mathfrak{u}(m)}+\widetilde{\omega}_{\mathfrak{u}(n)}\right\rangle}
\end{aligned}
$$

where $\Phi_{\mathfrak{k}}^{+}$is the set of all positive roots for $\mathfrak{k}$. Note that

$$
\operatorname{vol}_{\mathrm{HS}}\left(T_{(m)} \otimes T_{(n)}\right)=(2 \pi)^{m+n-1}
$$

since $\operatorname{dim}\left(\mathfrak{t}_{(m)} \otimes \mathbb{1}_{n}+\mathbb{1}_{m} \otimes \mathfrak{t}_{(n)}\right)=m+n-1$. Following the conventions in Example 3.3, we have

$$
\begin{aligned}
& \left\langle\tilde{\alpha}_{i j}^{(m)}, \widetilde{\omega}_{\mathfrak{u}(m)}+\widetilde{\omega}_{\mathfrak{u}(n)}\right\rangle=\left\langle\alpha_{i j}^{(m)} \otimes \tau_{n}, \omega_{\mathfrak{u}(m)} \otimes \tau_{n}\right\rangle+\left\langle\alpha_{i j}^{(m)} \otimes \tau_{n}, \tau_{m} \otimes \boldsymbol{\omega}_{\mathfrak{u}(n)}\right\rangle \\
& =\left\langle\alpha_{i j}^{(m)}, \omega_{\mathfrak{u}(m)}\right\rangle\left\langle\tau_{n}, \tau_{n}\right\rangle+\left\langle\alpha_{i j}^{(m)}, \tau_{m}\right\rangle\left\langle\tau_{n}, \omega_{\mathfrak{u}(n)}\right\rangle,
\end{aligned}
$$

that is,

$$
\left\langle\tilde{\alpha}_{i j}^{(m)}, \widetilde{\omega}_{\mathfrak{u}(m)}+\widetilde{\omega}_{\mathfrak{u}(n)}\right\rangle=\frac{j-i}{n}
$$

Similarly, we have

$$
\left\langle\tilde{\alpha}_{k l}^{(n)}, \widetilde{\omega}_{\mathfrak{u}(m)}+\widetilde{\omega}_{\mathfrak{u}(n)}\right\rangle=\frac{l-k}{m} .
$$

Furthermore,

$$
\begin{aligned}
\prod_{\alpha^{(m)} \in \Phi_{\mathfrak{u}(m)}^{+}} \frac{2 \pi}{\left\langle\tilde{\alpha}^{(m)}, \widetilde{\omega}_{\mathfrak{u}(m)}+\widetilde{\omega}_{\mathfrak{u}(n)}\right\rangle} & =\prod_{1 \leqslant i<j \leqslant m} \frac{2 \pi}{\left\langle\tilde{\alpha}_{i j}^{(m)}, \widetilde{\omega}_{\mathfrak{u}(m)}+\widetilde{\omega}_{\mathfrak{u}(n)}\right\rangle} \\
& =\prod_{1 \leqslant i<j \leqslant m} \frac{2 n \pi}{j-i}=n^{\left(\begin{array}{c}
m \\
2
\end{array}\right)} \operatorname{vol}_{\mathrm{HS}}\left(\mathrm{U}(m) / T_{(m)}\right)
\end{aligned}
$$


and

$$
\begin{aligned}
\prod_{\alpha^{(n)} \in \Phi_{\mathfrak{u}(n)}^{+}} \frac{2 \pi}{\left\langle\tilde{\alpha}^{(n)}, \widetilde{\omega}_{\mathfrak{u}(m)}+\widetilde{\omega}_{\mathfrak{u}(n)}\right\rangle} & =\prod_{1 \leqslant k<l \leqslant n} \frac{2 \pi}{\left\langle\tilde{\alpha}_{k l}^{(n)}, \widetilde{\omega}_{\mathfrak{u}(m)}+\widetilde{\omega}_{\mathfrak{u}(n)}\right\rangle} \\
& =\prod_{1 \leqslant k<l \leqslant n} \frac{2 m \pi}{l-k}=m^{\left(\begin{array}{c}
n \\
2
\end{array}\right)} \operatorname{vol}_{\mathrm{HS}}\left(\mathrm{U}(n) / T_{(n)}\right) .
\end{aligned}
$$

Therefore we can draw the following conclusion:

Theorem 3.5. For positive integers $m$ and $n$ greater than 1 , we have the following volume formulas:

$$
\begin{aligned}
& \operatorname{vol}_{\mathrm{HS}}\left(\mathrm{U}(m) \otimes \mathrm{U}(n) / T_{(m)} \otimes T_{(n)}\right)=m^{\left(\begin{array}{c}
n \\
2
\end{array}\right)} n^{\left(\begin{array}{c}
m \\
2
\end{array}\right)} \operatorname{vol}_{\mathrm{HS}}\left(\mathrm{U}(m) / T_{(m)}\right) \operatorname{vol}_{\mathrm{HS}}\left(\mathrm{U}(n) / T_{(n)}\right) \\
& =m^{\left(\begin{array}{c}
n \\
2
\end{array}\right)} n^{\left(\begin{array}{c}
m \\
2
\end{array}\right)} \frac{(2 \pi)^{\left(\begin{array}{c}
m \\
2
\end{array}\right)+\left(\begin{array}{c}
n \\
2
\end{array}\right)}}{\prod_{i=1}^{m} \Gamma(i) \prod_{j=1}^{n} \Gamma(j)}, \\
& \operatorname{vol}_{\mathrm{HS}}\left(T_{(m)} \otimes T_{(n)}\right)=(2 \pi)^{m+n-1}, \\
& \operatorname{vol}_{\mathrm{HS}}(\mathrm{U}(m) \otimes \mathrm{U}(n))=m^{\left(\begin{array}{c}
n \\
2
\end{array}\right)} n^{\left(\begin{array}{c}
m \\
2
\end{array}\right)} \frac{(2 \pi)^{\left(\begin{array}{c}
m+1 \\
2
\end{array}\right)+\left(\begin{array}{c}
n+1 \\
2
\end{array}\right)-1}}{\prod_{i=1}^{m} \Gamma(i) \prod_{j=1}^{n} \Gamma(j)} .
\end{aligned}
$$

In particular, for $(m, n)=(2,2)$, we have

$$
\operatorname{vol}_{\mathrm{HS}}(\mathrm{U}(2) \otimes \mathrm{U}(2))=128 \pi^{5} .
$$

\subsection{Symplectic volume}

The relation between flag manifolds and coadjoint orbits is well-known. To wit, let $\lambda \in \sqrt{-1} t^{*} \simeq \mathbb{R}^{n}$ and assume that $\lambda$ is dominant and regular under the coadjoint action. Let $\mathcal{O}_{K, \lambda}$ denote the orbit $\lambda$. Then there is a $K$-equivariantly diffeomorphism

$$
\mathcal{O}_{K, \lambda} \simeq K / T
$$

Moreover, $\mathcal{O}_{K, \lambda}$ has a standard symplectic form known as the Kirillov-Kostant-Souriau form. The following proposition gives a formula for the symplectic volume of $\mathcal{O}_{K, \lambda}$ (for a proof, see [5, Prop. 7.26]):

Proposition 3.6. Let $K$ be a compact connected Lie group of which $T$ is a maximal torus. Let $\lambda$ be a dominant vector in $\sqrt{-1} t^{*}$ that is a regular point under the coadjoint action. Let $\mathcal{O}_{K, \lambda}$ be the orbit through $\lambda$. The symplectic volume of $\mathcal{O}_{K, \lambda}$ relative to the standard symplectic form is

$$
\operatorname{vol}_{\text {symp }}\left(\mathcal{O}_{K, \lambda}\right)=\prod_{\alpha \in \Phi_{\mathfrak{k}}^{+}} \frac{\langle\lambda, \alpha\rangle}{\langle\omega, \alpha\rangle} .
$$

Here $\omega=\frac{1}{2} \sum_{\alpha \in \Phi_{\mathfrak{e}}^{+}} \alpha$.

Example 3.7. Consider the unitary group $\mathrm{U}(n)$. Using the Hilbert-Schmidt inner product, we can identify $\mathfrak{u}(n)^{*}$ and $\mathfrak{u}(n)$; since the Hilbert-Schmidt inner product is invariant under the adjoint action, we can also identify adjoint and coadjoint orbits. So we may speak of the symplectic volume of the adjoint orbit $\mathcal{O}_{\Lambda}$ of $\Lambda \in \sqrt{-1} \mathfrak{t}$ where $\mathfrak{t}$ is the standard maximal toral subalgebra of $\mathfrak{u}(n)$, that is, the set of diagonal matrices in $\mathfrak{u}(n)$. Note that

$$
\mathcal{O}_{\Lambda}=\operatorname{Ad}(\mathrm{U}(n)) \Lambda=\left\{U \Lambda U^{*}: \Lambda=\operatorname{diag}\left(\lambda_{1}, \ldots, \lambda_{n}\right) \text { with } \lambda_{i} \in \mathbb{R} \text { for all } i=1, \ldots, n \text { and } \lambda_{1}>\cdots>\lambda_{n}\right\} .
$$


Continuing with the conventions set up in Example 3.3, we have, by Equation (3.29),

$$
\operatorname{vol}_{\text {symp }}\left(\mathcal{O}_{\Lambda}\right)=\prod_{i<j} \frac{\left\langle\alpha_{i j}, \lambda\right\rangle}{\left\langle\alpha_{i j}, \omega\right\rangle}=\prod_{i<j} \frac{\lambda_{i}-\lambda_{j}}{j-i}=\frac{\prod_{i<j}\left(\lambda_{i}-\lambda_{j}\right)}{1 ! \cdots(n-1) !}=\frac{\prod_{i<j}\left(\lambda_{i}-\lambda_{j}\right)}{\prod_{j=1}^{n} \Gamma(j)} .
$$

This result appears in [7, Eq. (2.3)].

Theorem 3.8. Let $K=\mathrm{U}(m) \otimes \mathrm{U}(n)$ and $T=T_{(m)} \otimes T_{(n)}$ as stated at the beginning of this section. Let $\Lambda=\operatorname{diag}\left(\lambda_{1,1}, \ldots, \lambda_{m, 1}, \ldots, \lambda_{1, n}, \ldots, \lambda_{m, n}\right)$ where $\lambda_{i, j}$ 's are real numbers satisfying $\lambda_{1,1}>\ldots>\lambda_{m, n}$. Then $\Lambda$ is a regular point in the maximal toral subalgebra $\sqrt{-1} \mathfrak{t}_{(m n)}$ of $\sqrt{-1} \mathfrak{u}(\mathrm{mn})$. Let $\lambda$ be a regular point in $\sqrt{-1} \mathrm{t}$ derived from $\Lambda$, i.e., $\lambda=\operatorname{Tr}_{n}(\Lambda) \otimes \mathbb{1}_{n}+\mathbb{1}_{m} \otimes \operatorname{Tr}_{m}(\Lambda)$. Then the symplectic volume of the adjoint orbit $\mathcal{O}_{\lambda}$ is given by

$$
\begin{aligned}
\operatorname{vol}_{\text {symp }}\left(\mathcal{O}_{\lambda}\right) & =\operatorname{vol}_{\text {symp }}\left(\mathcal{O}_{\operatorname{Tr}_{m}(\Lambda)}\right) \operatorname{vol}_{\text {symp }}\left(\mathcal{O}_{\operatorname{Tr}_{n}(\Lambda)}\right) \\
& =\frac{\left[\prod_{1 \leqslant i<j \leqslant m} \sum_{k=1}^{n}\left(\lambda_{i k}-\lambda_{j k}\right)\right]\left[\prod_{1 \leqslant i<j \leqslant n} \sum_{i=1}^{m}\left(\lambda_{i k}-\lambda_{i l}\right)\right]}{\prod_{i=1}^{m} \Gamma(i) \prod_{k=1}^{n} \Gamma(k)} .
\end{aligned}
$$

Proof. Thanks to Propositions 3.4 and 3.6, we have

$$
\operatorname{vol}_{\text {symp }}\left(\mathcal{O}_{\lambda}\right)=\prod_{\alpha^{(m)} \in \Phi_{\mathfrak{u}(m)}^{+}} \frac{\left\langle\tilde{\alpha}^{(m)}, \lambda\right\rangle}{\left\langle\tilde{\alpha}^{(m)}, \widetilde{\boldsymbol{\omega}}_{\mathfrak{u}(m)}+\widetilde{\omega}_{\mathfrak{u}(n)}\right\rangle} \prod_{\alpha^{(n)} \in \Phi_{\mathfrak{u}(n)}^{+}} \frac{\left\langle\tilde{\alpha}^{(n)}, \lambda\right\rangle}{\left\langle\tilde{\alpha}^{(n)}, \widetilde{\omega}_{\mathfrak{u}(m)}+\widetilde{\boldsymbol{\omega}}_{\mathfrak{u}(n)}\right\rangle} .
$$

Continuing with the conventions used in Example 3.7, wea hve

$$
\operatorname{vol}_{\text {symp }}\left(\mathcal{O}_{\lambda}\right)=\prod_{1 \leqslant i<j \leqslant m} \frac{\left\langle\tilde{\alpha}_{i j}^{(m)}, \lambda\right\rangle}{\left\langle\tilde{\alpha}_{i j}^{(m)}, \widetilde{\omega}_{\mathfrak{u}(m)}+\widetilde{\omega}_{\mathfrak{u}(n)}\right\rangle} \prod_{1 \leqslant i<j \leqslant n} \frac{\left\langle\tilde{\alpha}_{k l}^{(n)}, \lambda\right\rangle}{\left\langle\tilde{\alpha}_{k l}^{(n)}, \widetilde{\omega}_{\mathfrak{u}(m)}+\widetilde{\omega}_{\mathfrak{u}(n)}\right\rangle} .
$$

Since

$$
\left\langle\tilde{\alpha}_{i j}^{(m)}, \lambda\right\rangle=\left\langle\alpha_{i j}^{(m)} \otimes \tau_{n}, \lambda\right\rangle=\frac{1}{n} \operatorname{Tr}\left(\left(\left(E_{i i}-E_{j j}\right) \otimes \mathbb{1}_{n}\right) \Lambda\right)=\frac{1}{n} \operatorname{Tr}\left(\left(E_{i i}-E_{j j}\right) \operatorname{Tr}_{n}(\Lambda)\right)
$$

and

$$
\left\langle\tilde{\alpha}_{k l}^{(n)}, \lambda\right\rangle=\left\langle\tau_{m} \otimes \alpha_{k l}^{(n)}, \lambda\right\rangle=\frac{1}{m} \operatorname{Tr}\left(\left(\mathbb{1}_{m} \otimes\left(E_{k k}-E_{l l}\right)\right) \Lambda\right)=\frac{1}{m} \operatorname{Tr}\left(\left(E_{k k}-E_{l l}\right) \operatorname{Tr}_{m}(\Lambda)\right)
$$

it follows that

$$
\left\langle\tilde{\alpha}_{i j}^{(m)}, \lambda\right\rangle=\frac{1}{n} \sum_{k=1}^{n}\left(\lambda_{i k}-\lambda_{j k}\right)
$$

and

$$
\left\langle\tilde{\alpha}_{k l}^{(n)}, \lambda\right\rangle=\frac{1}{m} \sum_{i=1}^{m}\left(\lambda_{i k}-\lambda_{i l}\right)
$$

Therefore,

$$
\begin{aligned}
\operatorname{vol}_{\text {symp }}\left(\mathcal{O}_{\lambda}\right) & =\prod_{1 \leqslant i<j \leqslant m} \frac{\frac{1}{n} \sum_{k=1}^{n}\left(\lambda_{i k}-\lambda_{j k}\right)}{\frac{1}{n}(j-i)} \prod_{1 \leqslant i<j \leqslant n} \frac{\frac{1}{m} \sum_{i=1}^{m}\left(\lambda_{i k}-\lambda_{i l}\right)}{\frac{1}{m}(l-k)} \\
& =\prod_{1 \leqslant i<j \leqslant m} \frac{\sum_{k=1}^{n}\left(\lambda_{i k}-\lambda_{j k}\right)}{j-i} \prod_{1 \leqslant i<j \leqslant n} \frac{\sum_{i=1}^{m}\left(\lambda_{i k}-\lambda_{i l}\right)}{l-k} .
\end{aligned}
$$

Together with (3.30), we have the desired equalities (3.32) and (3.31). 
Corollary 3.9. Let $K=\mathrm{U}(2) \otimes \mathrm{U}(2)$ and $T=T_{(2)} \otimes T_{(2)}$. Let $\Lambda=\operatorname{diag}\left(\lambda_{1}, \lambda_{2}, \lambda_{3}, \lambda_{4}\right) \in \sqrt{-1} \mathrm{t}$ with $\lambda_{1}>\lambda_{2}>\lambda_{3}>\lambda_{4} \geqslant 0$ and $\sum_{j=1}^{4} \lambda_{j}=1$. Let $\mathcal{O}_{\lambda}^{\mathrm{LU}}$ denote the adjoint orbit of $\Lambda$, that is,

$$
\mathcal{O}_{\Lambda}^{\mathrm{LU}}:=\left\{(U \otimes V) \Lambda\left(U^{-1} \otimes V^{-1}\right): U, V \in \mathrm{U}(2)\right\} .
$$

Then the symplectic volume of such local unitary orbit is given by

$$
\operatorname{vol}_{\text {symp }}\left(\mathcal{O}_{\lambda}^{\mathrm{LU}}\right)=\left(\lambda_{1}+\lambda_{2}-\lambda_{3}-\lambda_{4}\right)\left(\lambda_{1}+\lambda_{3}-\lambda_{2}-\lambda_{4}\right) .
$$

Proof. This is a direct consequence of Theorem 3.8.

Remark 3.10. Let $\Lambda$ be as in Corollary 3.9, and let

$$
\mathcal{O}_{\Lambda}^{\mathrm{GU}}:=\left\{W \Lambda W^{-1}: W \in \mathrm{U}(4)\right\}
$$

By (3.30), we have

$$
\operatorname{vol}_{\text {symp }}\left(\mathcal{O}_{\Lambda}^{\mathrm{GU}}\right)=\frac{1}{12} \prod_{1 \leqslant i<j \leqslant 4}\left(\lambda_{i}-\lambda_{j}\right)
$$

Meanwhile, under the constraint $\lambda_{1}>\lambda_{2}>\lambda_{3}>\lambda_{4} \geqslant 0$ and $\sum_{j=1}^{4} \lambda_{j}=1$, we have

$$
\begin{aligned}
& \operatorname{vol}_{\text {symp }}\left(\mathcal{O}_{\lambda}^{\mathrm{LU}}\right)=\left[\left(\lambda_{1}-\lambda_{3}\right)+\left(\lambda_{2}-\lambda_{4}\right)\right]\left[\left(\lambda_{1}-\lambda_{2}\right)+\left(\lambda_{3}-\lambda_{4}\right)\right] \\
& >4 \sqrt{\left(\lambda_{1}-\lambda_{3}\right)\left(\lambda_{2}-\lambda_{4}\right)\left(\lambda_{1}-\lambda_{2}\right)\left(\lambda_{3}-\lambda_{4}\right)} \\
& >4\left(\lambda_{1}-\lambda_{3}\right)\left(\lambda_{2}-\lambda_{4}\right)\left(\lambda_{1}-\lambda_{2}\right)\left(\lambda_{3}-\lambda_{4}\right) \\
& >4 \prod_{1 \leqslant i<j \leqslant 4}\left(\lambda_{i}-\lambda_{j}\right)>48 \operatorname{vol}_{\text {symp }}\left(\mathcal{O}_{\Lambda}^{\mathrm{GU}}\right)>\operatorname{vol}_{\text {symp }}\left(\mathcal{O}_{\Lambda}^{\mathrm{GU}}\right) .
\end{aligned}
$$

So we see that $\operatorname{vol}_{\text {symp }}\left(\mathcal{O}_{\Lambda}^{\mathrm{GU}}\right)<\operatorname{vol}_{\text {symp }}\left(\mathcal{O}_{\lambda}^{\mathrm{LU}}\right)$. At the same time, $\mathcal{O}_{\Lambda}^{\mathrm{LU}}$ is a submanifold of $\mathcal{O}_{\Lambda}^{\mathrm{GU}}$ since $\mathrm{U}(2) \otimes \mathrm{U}(2)$ is a Lie subgroup of $\mathrm{U}(4)$; but this is not a contradiction because the measures for $\mathcal{O}_{\Lambda}^{\mathrm{LU}}$ and $\mathcal{O}_{\Lambda}^{\mathrm{GU}}$ have no a priori relation, so one cannot directly compare the two. In fact, $\mathcal{O}_{\Lambda}^{\mathrm{LU}}$ is a set of zero-measure in $\mathcal{O}_{\Lambda}^{\mathrm{GU}}$ because $\operatorname{dim}\left(\mathcal{O}_{\Lambda}^{\mathrm{LU}}\right)<\operatorname{dim}\left(\mathcal{O}_{\Lambda}^{\mathrm{GU}}\right)$.

\section{Discussion and concluding remarks}

There may exist many $\Lambda$ 's corresponding to a single $\lambda$ in Theorem 3.8. A relevant well-known problem is the so-called quantum marginal problem. For the two-qubit system, there is a nice solution for it [6]. Specifically, mixed two-qubit state $\rho_{A B}$ with spectrum $\lambda_{1} \geqslant \lambda_{2} \geqslant \lambda_{3} \geqslant \lambda_{4} \geqslant 0$ and margins $\rho_{A}$ and $\rho_{B}$ exists if and only if minimal eigenvalues $\lambda_{A}$ and $\lambda_{B}$ of the margins satisfy the following inequalities:

$$
\left\{\begin{array}{l}
\min \left(\lambda_{A}, \lambda_{B}\right) \geqslant \lambda_{3}+\lambda_{4}, \\
\lambda_{A}+\lambda_{B} \geqslant \lambda_{2}+2 \lambda_{3}+\lambda_{4}, \\
\left|\lambda_{A}-\lambda_{B}\right| \leqslant \min \left(\lambda_{1}-\lambda_{3}, \lambda_{2}-\lambda_{4}\right) .
\end{array}\right.
$$

Here we examine a specific example showing this property. Let $\rho_{A B}$ be any two-qudit in $\mathrm{D}\left(\mathbb{C}^{d} \otimes \mathbb{C}^{d}\right)$. Then there exists a global unitary $V \in \mathrm{U}\left(d^{2}\right)$ such that $\rho_{A B}^{\prime}=V \rho_{A B} V^{\dagger}$ with two marginal states as 
$\rho_{A}^{\prime}=\rho_{B}^{\prime}=\mathbb{1}_{d} / d$. Indeed, by the Spectral Decomposition Theorem, we have the following decomposition: Writing $[k]:=\{1, \ldots, k\}$ for any positive integer $k$, we have

$$
\rho_{A B}=\sum_{j=1}^{d^{2}} \lambda_{j}\left|\Psi_{j}\right\rangle\left\langle\Psi_{j}|, \quad| \Psi_{j}\right\rangle \in \mathbb{C}^{d} \otimes \mathbb{C}^{d},
$$

where $\lambda_{j} \geqslant 0$ for each $j \in\left[d^{2}\right]$, and $\left\{\left|\Psi_{j}\right\rangle: j \in\left[d^{2}\right]\right\}$ are the eigenvectors corresponding to eigenvalues $\lambda_{j}$. There exists a collection of unitary matrices, called discrete Weyl unitary matrices, $W_{j} \in \mathrm{U}(d), j \in\left[d^{2}\right]$, that form a unitary matrix basis for $M_{d}(\mathbb{C})$, the set of all $d \times d$ complex matrices. If we denote by $\operatorname{vec}(M)$ is the vectorization of a complex rectangular matrix $M$, that is, $\operatorname{vec}(M):=\sum_{i, j} M_{i j}|i j\rangle$ where $M=\sum_{i, j} M_{i j}|i\rangle\langle j|$, then $\left\{\operatorname{vec}\left(W_{j}\right): j \in\left[d^{2}\right]\right\}$ forms a maximally entangled basis for $\mathbb{C}^{d} \otimes \mathbb{C}^{d}$ (see also for its generalization in [9]). So there is a global unitary matrix $V \in \mathrm{U}\left(d^{2}\right)$ such that

$$
V\left|\Psi_{j}\right\rangle=\frac{1}{\sqrt{d}} \operatorname{vec}\left(W_{j}\right), j \in\left[d^{2}\right],
$$

since $\left\{\left|\Psi_{j}\right\rangle: j \in\left[d^{2}\right]\right\}$ and $\left\{\frac{1}{\sqrt{d}} \operatorname{vec}\left(W_{j}\right): j \in\left[d^{2}\right]\right\}$ are two orthonormal bases for the same space $\mathbb{C}^{d} \otimes \mathbb{C}^{d}$. This implies that

$$
\rho_{A B}^{\prime}=V \rho_{A B} V^{\dagger}=\sum_{j=1}^{d^{2}} \lambda_{j} V\left|\Psi_{j}\right\rangle\left\langle\Psi_{j}\right| V^{\dagger}=\frac{1}{d} \sum_{j=1}^{d^{2}} \lambda_{j} \operatorname{vec}\left(W_{j}\right) \operatorname{vec}\left(W_{j}\right)^{\dagger} .
$$

Hence,

$$
\rho_{A}^{\prime}=\frac{1}{d} \sum_{j=1}^{d^{2}} \lambda_{j} W_{j} W_{j}^{+}=\frac{\mathbb{1}_{d}}{d} \sum_{j=1}^{d^{2}} \lambda_{j}=\frac{\mathbb{1}_{d}}{d} \operatorname{Tr}\left(\rho_{A B}\right)=\frac{\mathbb{1}_{d}}{d}
$$

and

$$
\rho_{B}^{\prime}=\frac{1}{d} \sum_{j=1}^{d^{2}} \lambda_{j}\left(W_{j}^{\dagger} W_{j}\right)^{\top}=\frac{\mathbb{1}_{d}}{d} \sum_{j=1}^{d^{2}} \lambda_{j}=\frac{\mathbb{1}_{d}}{d} \operatorname{Tr}\left(\rho_{A B}\right)=\frac{\mathbb{1}_{d}}{d} .
$$

Therefore, $\rho_{A}^{\prime}=\rho_{B}^{\prime}=\mathbb{1}_{d} / d$. This example also indicates that the maximum of mutual information along a global unitary orbit of a given bipartite state with the prescribed spectrum $\Lambda$ is $2 \ln (d)-S(\Lambda)$ [11, 12], where $S(\Lambda)$ is the von Neumann entropy. Now fix $\rho_{A}$ and $\rho_{B}$, and denote by $\mathcal{C}\left(\rho_{A}, \rho_{B}\right)$ the set of all bipartite states $\rho_{A B}$ with fixed marginal states $\rho_{A}$ and $\rho_{B}$, respectively. It is known that $\mathcal{C}\left(\rho_{A}, \rho_{B}\right)$ is a compact convex set. Moreover, in Parthasarathy [18], a necessary and sufficient condition is presented for an element $\rho_{A B}$ in $\mathcal{C}\left(\rho_{A}, \rho_{B}\right)$ to be an extreme point; in the two-qubit case, the condition amounts to a two-qubit state $\rho_{A B} \in \mathcal{C}(\mathbb{1} / 2, \mathbb{1} / 2)$ being maximally entangled.

There are several open questions which are presented below:

1. Suppose that bipartite states $\rho$ and $\tilde{\rho}$ are not LU equivalent, while $\rho$ is locally diagonalizable but $\tilde{\rho}$ is not. As we have seen previously, the HS volume of $\mathcal{O}_{\rho}^{\mathrm{LU}}$ can be calculated. The question is how to calculate the HS volume of $\mathcal{O}_{\tilde{\rho}}^{\mathrm{LU}}$ ?

2. It is easily seen that $\mathscr{D}_{\mathrm{LU}}\left(\mathbb{C}^{m} \otimes \mathbb{C}^{n}\right)$ can be partitioned into local unitary orbits, that is,

$$
\mathscr{D}_{\mathrm{LU}}\left(\mathbb{C}^{m} \otimes \mathbb{C}^{n}\right)=\bigsqcup_{\Lambda \in \Delta_{m n-1}} \mathcal{O}_{\Lambda}^{\mathrm{LU}}
$$


How do we get the HS volume $\operatorname{vol}_{\mathrm{HS}}\left(\mathscr{D}_{\mathrm{LU}}\left(\mathbb{C}^{m} \otimes \mathbb{C}^{n}\right)\right)$ from the HS volumes $\operatorname{vol}_{\mathrm{HS}}\left(\mathcal{O}_{\Lambda}^{\mathrm{LU}}\right)$ of local unitary orbits $\mathcal{O}_{\Lambda}^{\mathrm{LU}}$ ?

3. Can we establish some kind of a "canonical form" for bipartite states like the Singular Value Decomposition (SVD) for complex matrices and/or the Spectral Decomposition for normal matrices, especially for any two-qubit states? Also, how to obtain that?

4. Because $\mathcal{C}\left(\rho_{A}, \rho_{B}\right)$ is a compact convex set, its HS volume can in principle be calculated. To the best of our knowledge, an analytical expression for its HS volume has not been founded. We note that the volume for $\mathcal{C}(\mathbb{1} / 2, \mathbb{1} / 2)$ relative to the Lebesgue measure can be found in [14]: $\operatorname{vol}_{g}(\mathcal{C}(\mathbb{1} / 2, \mathbb{1} / 2))=$ $2 \pi^{4} / 315$. Note also that we can identify $\mathcal{C}(1 / 2,1 / 2)$ with the set of all unital qubit quantum channels via Choi-Jamiłkowski isomorphism. The problem of calculating the HS volume of $\mathcal{C}(1 / 2,1 / 2)$ and some relevant discussions can also be found in [20].

In summary, we analyzed in this paper the geometry of locally diagonalizable bipartite states its Hilbert-Schmidt volume and symplectic volume. We obtained an expression for the HS volume involving an integral, so in principle, we could work out an analytical formula; but for now, we evaluated the volume in the two-qubit case. In addition, we obtained a necessary and sufficient condition for a twoqubit state to be LU equivalent to a diagonal state. A generalization to higher dimensional cases is still open. After introducing Harish-Chandra's volume formula for flag manifolds, we turned to the geometry of local unitary orbits. We found that Harish-Chandra's volume formula can be applied to calculate the volume of local unitary orbits. We also obtained a volume formula for the tensor product $\mathbf{U}(m) \otimes \mathbf{U}(n)$. Although this is a direct consequence of Harish-Chandra's volume formula, there is, to our knowledge, no record of it in the literature. We believe these results and the questions raised can shed new lights and spur relevant research in quantum information theory.

\section{Acknowledgements}

This research was supported by Zhejiang Provincial Natural Science Foundation of China under Grant No. LY17A010027 and NSFC (Nos.11301124, 61771174), and also supported by the crossdisciplinary innovation team building project of Hangzhou Dianzi University. SH was partially supported under Northwestern Scholarship Grant.

\section{References}

[1] A. Andai, Volume of the quantum mechanical state space, J. Phys. A : Math. Theor. 39, 13641 (2006).

[2] G. Aubrun and S.J. Szarek, Tensor products of convex sets and the volume of separable states on $n$ qubits, Phys. Rev. A 73(2), 022109 (2006).

[3] G. Aubrun, S.J. Szarek and D. Ye, Entanglement thresholds for random induced states, Commun. Pure Appl. Math. 67(1), 1-52 (2014).

[4] I. Bengtsson and K. Życzkowski, Geometry of quantum states: An Introduction to Quantum Entanglement, Cambridge University Press (2006). 
[5] N. Berline, E. Getzler and M. Vergne, Heat kernels and Dirac operators, Springer-Verlag, New York (1992).

[6] S. Bravyi, Compatibility between local and multipartite states, Quant Inf. \& Comput. 4(1), 012-026 (2004).

[7] M. Christandl, B. Doran, S. Kousidis, M. Walter, Eigenvalue distributions of reduced density matrices, Comm. Math. Phys. 332, 1-52 (2014).

[8] J.J. Duistermaat, J.A.C Kolk, Lie Groups, Springer-Verlage New York, Inc. (2000).

[9] Y. Guo, S. Du, X. Li, and S. Wu, Entangled bases with fixed Schmidt number, J. Phys. A : Math. Theor. 48, 245301 (2015).

[10] Harish-Chandra, Harmonic analysis on real reductive groups I: The theory of the constant term, J. Funct. Anal. 19, 104-204 (1975).

[11] S. Jevtic, D. Jennings, and T. Rudolph, Maximally and minimally correlated states attainable within a closed evolving system, Phys. Rev. Lett. 108, 110403 (2012).

[12] S. Jevtic, D. Jennings, and T. Rudolph, Quantum mutual information along unitary orbits, Phys. Rev. A 85, 052121 (2012).

[13] N. Jing, S-M. Fei, M. Li, X. Li-Jost, and T. Zhang, Local unitary invariants of generic multiqubit states, Phys. Rev. A 92, 022306 (2015).

[14] A. Lovas, A. Andai, Volume of the space of qubit channels and some new results about the distribution of the quantum Dobrushin coefficient, arXiv:1607.01215

[15] A. Lovas, A. Andai, Invariance of separability probability over reduced states in $4 \times 4$ bipartite systems, J. Phys. A : Math. Theor. 50, 295303 (2017).

[16] I.G. Macdonald, The volume of a compact Lie group, Invent. Math. 56, 93-95 (1980).

[17] S. Milz and W.T. Strunz, Volumes of conditioned bipartite state spaces, J. Phys. A : Math. Theor. 48, 035306 (2014).

[18] K.R. Parthasarathy, Extremal quantum states in coupled systems, Annales de l'Institut Henri Poincare (B) Probability and Statistics 41, 257-268 (2005).

[19] P.B. Slater and C.F. Dunkl, Moment-based evidence for simple rational-valued Hilbert-Schmidt generic $2 \times 2$ separability probabilities, J. Phys. A : Math. Theor. 45, 095305(2012).

[20] L. Zhang, Volumes of orthogonal groups and unitary groups, arXiv:1509.00537

[21] K. Życzkowski, P. Horodecki, A. Sanpera, and M. Lewenstein, Volume of the set of separable states, Phys. Rev. A 58, 883 (1998).

[22] K. Życzkowski and I. Bengtsson, On Duality between Quantum Maps and Quantum States, Open Systems \& Information Dynamics 11 3-42 (2004). 
[23] K. Życzkowski and H.J. Sommers, Induced measures in the space of mixed quantum states, J. Phys. A : Math. Gen. 34, 7111 (2001).

[24] K. Życzkowski and H.J. Sommers, Hilbert-Schmidt volume of the set of mixed quantum states, J. Phys. A : Math. Gen. 36, 10115 (2003). 